Supplementary Information for:

\title{
Assessment of Robustness to Temperature in a Negative Feedback Loop and a Feedforward Loop
}

Abhilash Patel ${ }^{1}$, Richard M. Murray², Shaunak Sen ${ }^{1}$ *

1Department of Electrical Engineering, Indian Institute of Technology Delhi, Hauz Khas, New Delhi 110016, India ${ }^{2}$ California Institute of Technology, Pasadena, California 91125, United States

*Email: shaunak.sen@ee.iitd.ac.in 


\section{Contents}

(A) Supplementary Experimental Data (Supplementary Figures 1-4)

(B) Steady State of the Negative Feedback Model (Supplementary Figure 5)

(C) Flowchart for Computing $Q_{x s s}$ (Supplementary Figure 6)

(D) Computational Assessment of Model Variants (Supplementary Figures 7-11)

(E) Computational Assessment of a Coherent Feedforward Loop Model (Supplementary Figures 12-13)

(F) Additional Data Analysis of Negative Feedback Loop Experiments (Supplementary Figures 14-15)

(G) Additional Data, Data Analysis, and Model for the Cell-Free Experiments (Supplementary Figures 16-19) 


\section{Supplementary Figure 1.}

(a) Optical density and (b) Fluorescence values of the data from the negative feedback circuit.
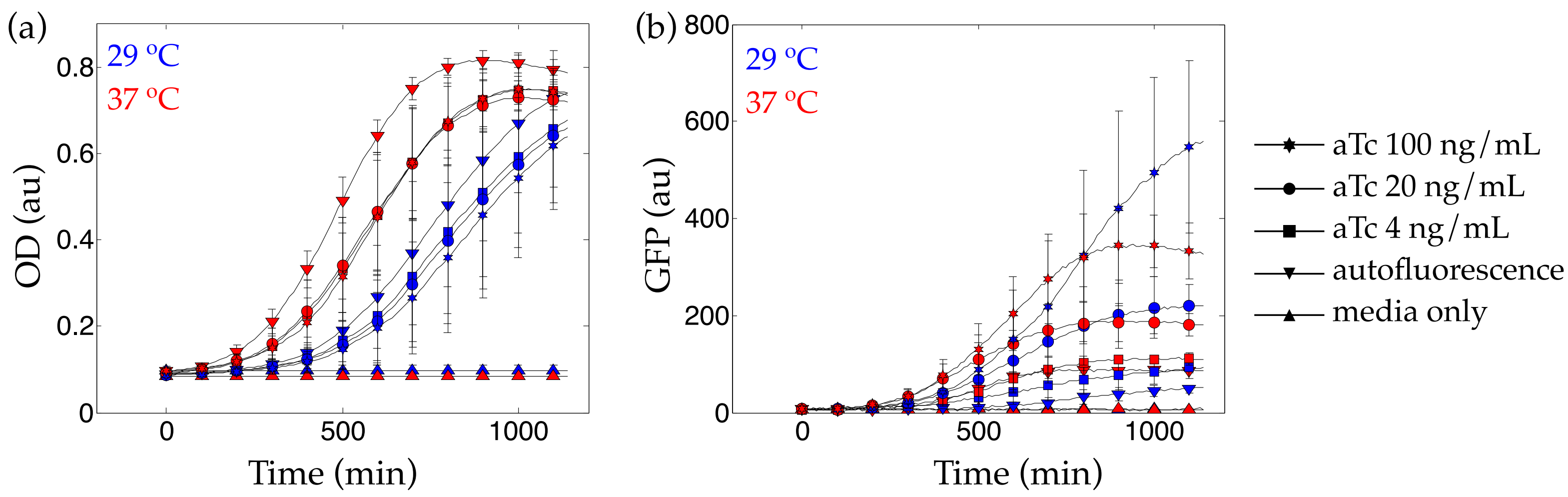


\section{Supplementary Figure 2.}

\section{Experimental assessment of negative feedback circuit.}

Blue and red lines are the measured responses at 29 and $37{ }^{\circ} \mathrm{C}$, respectively. Circles and dots indicate the response of the negative feedback loop and autofluorescence, respectively. The aTc concentrations are indicated.
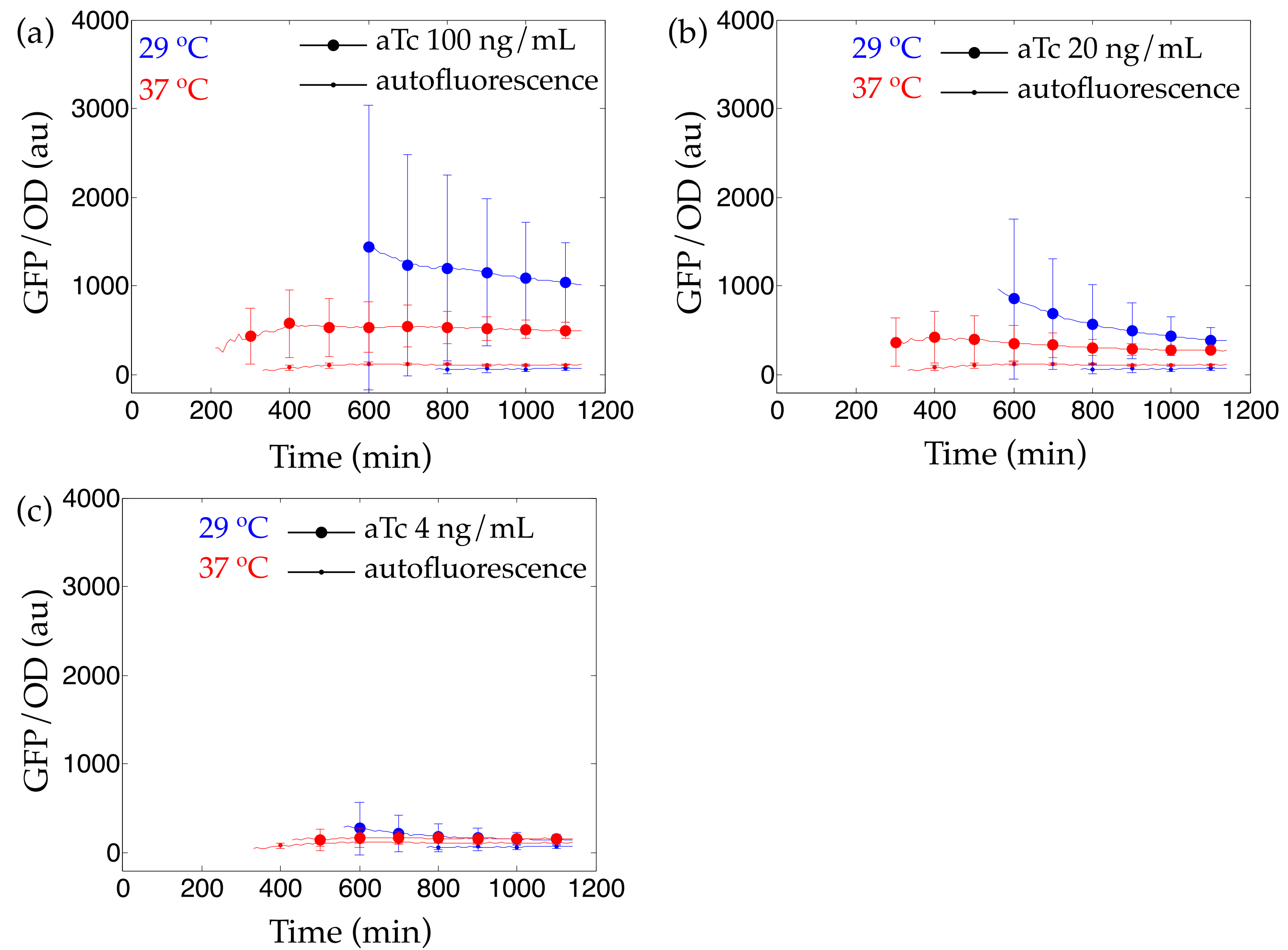


\section{Supplementary Figure 3.}

Temperature profiles for the experimental assessment of negative feedback. Solid blue lines correspond to the data that were plotted in Figure 2.

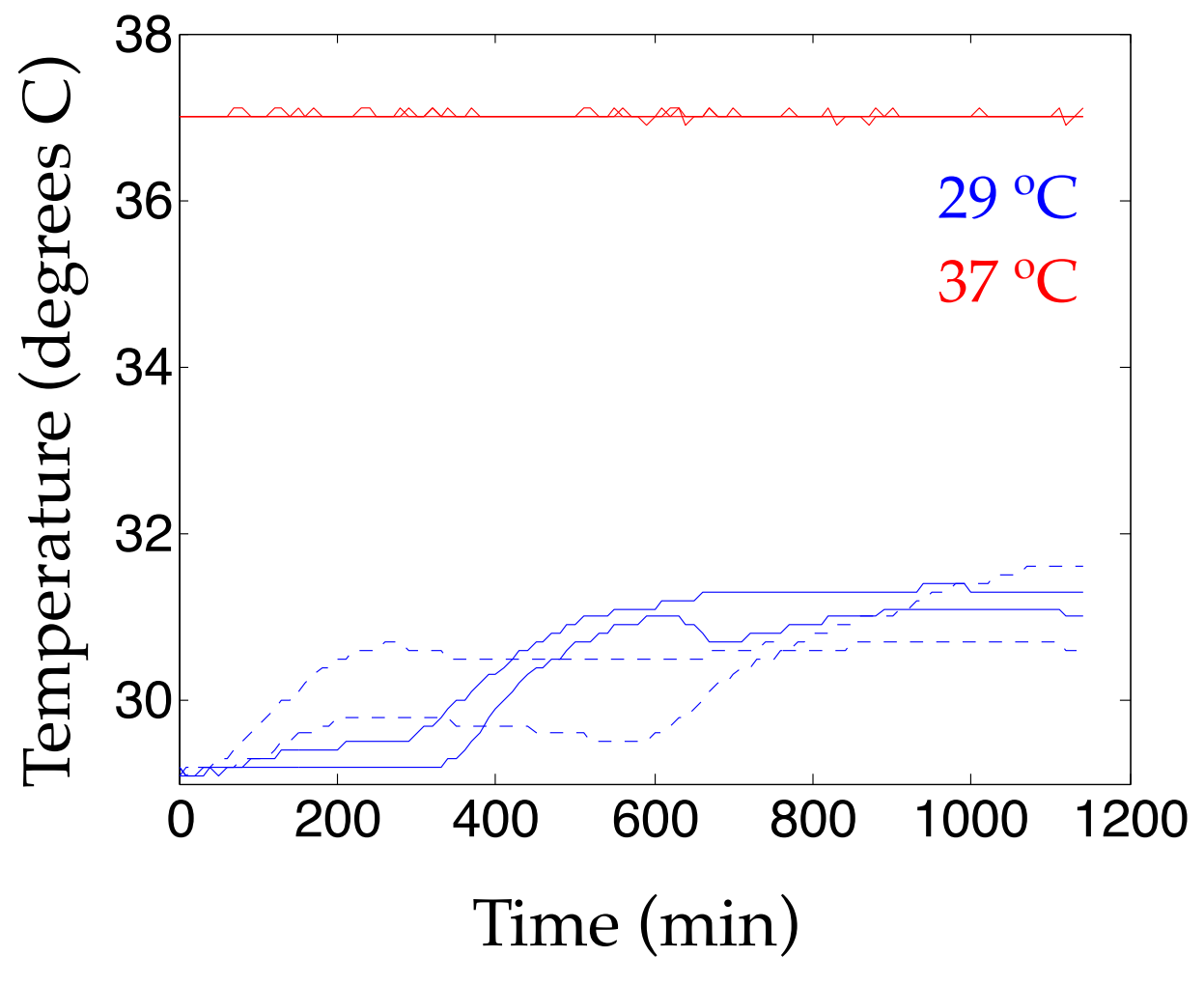




\section{Supplementary Figure 4.}

(a) Optical density, (b) Fluorescence, and (c) Optical density-normalized fluorescence values of the data from the feedforward loop circuit.
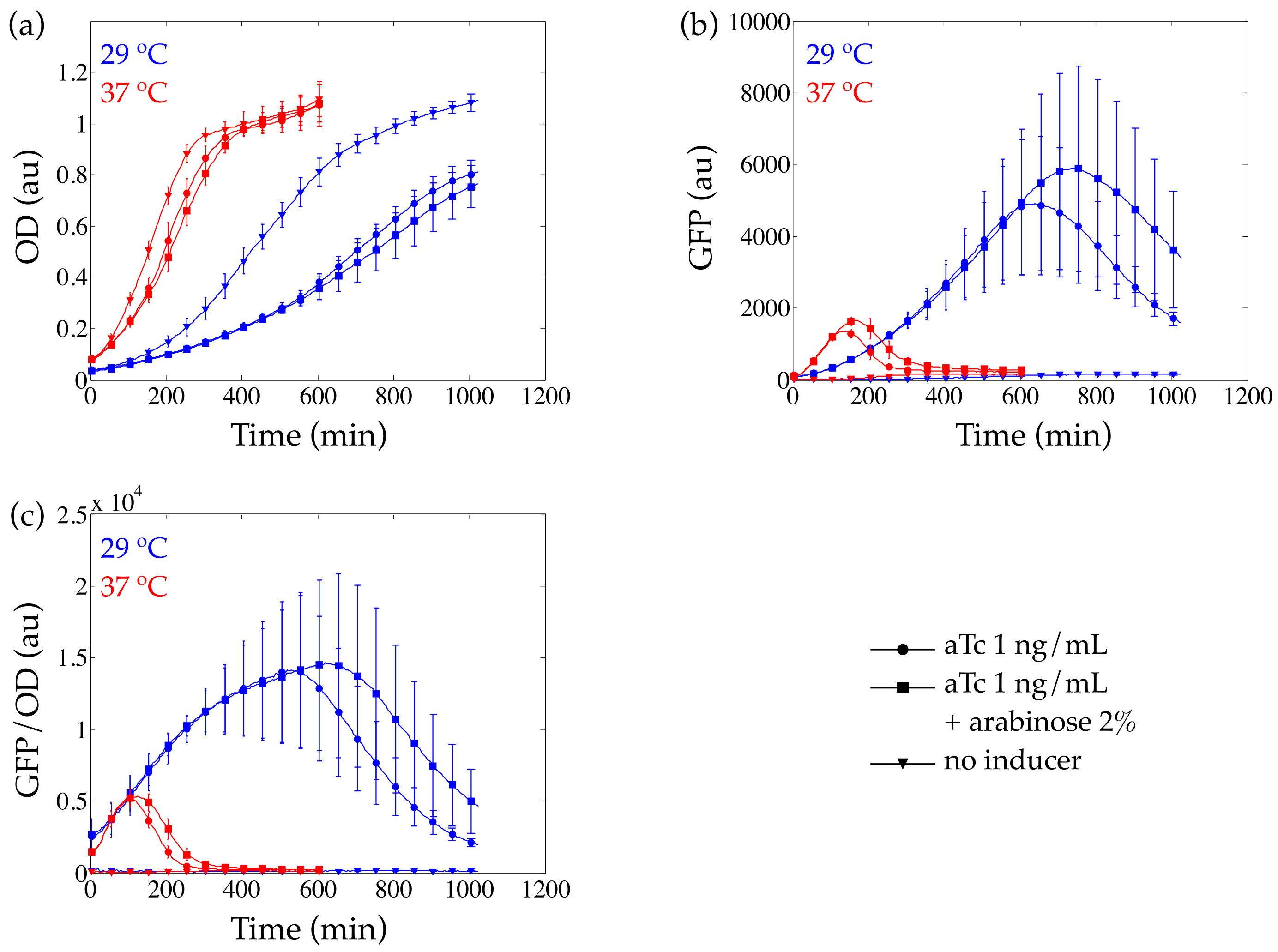

$\longrightarrow$ aTc $1 \mathrm{ng} / \mathrm{mL}$

$\longrightarrow$ - aTc $1 \mathrm{ng} / \mathrm{mL}$ + arabinose $2 \%$

$\longrightarrow$ no inducer 


\section{Supplementary Figure 5.}

Steady state of the negative feedback model. (a) Solid lines are plots of the production and the degradation terms of the model as a function of $x$. The production term is a decreasing function and the degradation term is an increasing function. The steady state is the abscissa of their point of intersection. This steady state is less than $\alpha / \gamma$, which is the abscissa of the point of intersection of a production term with constant rate $\alpha$ (dashed line) and the degradation term. The plots are for the default value of parameters considered in the main text. (b) The constant production rate is the limiting case of the production term that does not decrease. In terms of the parameters, this is the limit $k \gg \alpha / \gamma$. The limiting value of the steady state is illustrated algebraically below.

(a)

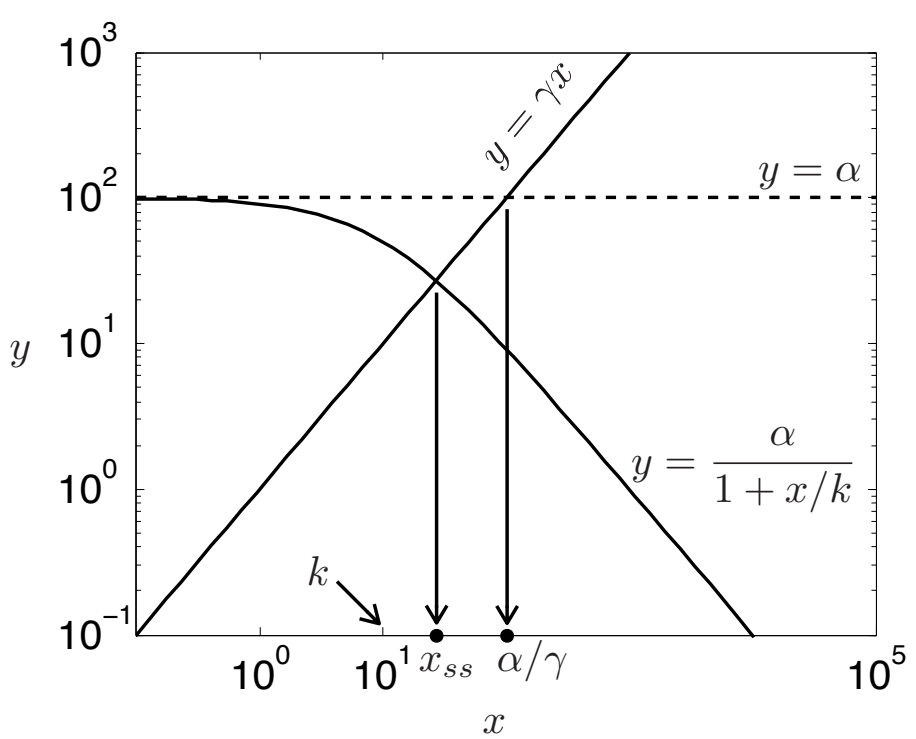

(b)

At steady state, $\frac{d x}{d t}=0, \Rightarrow \frac{\alpha}{1+x / k}-\gamma x=0$,

$\Rightarrow x^{2}+k x-\alpha k / \gamma=0, \Rightarrow x_{s s}=\frac{-k+\sqrt{k^{2}+4 \alpha k / \gamma}}{2}$.

Because $\sqrt{k^{2}+4 \alpha k / \gamma}>k$, only positive sign is considered.

For small $\frac{\alpha / \gamma}{k}$, the term with the square root is expanded

in a Taylor Series in powers of $\frac{\alpha / \gamma}{k}$.

$$
\begin{aligned}
x_{s s} & =\frac{k}{2}\left(-1+\sqrt{\left.1+4 \frac{\alpha / \gamma}{k}\right)},\right. \\
& =\frac{k}{2}\left(-1+1+\frac{1}{2} 4 \frac{\alpha / \gamma}{k}+\frac{1}{2} \frac{-1}{2^{2}} 4^{2}\left(\frac{\alpha / \gamma}{k}\right)^{2}+\cdots\right), \\
& =\frac{\alpha}{\gamma}\left(1+\text { terms containing } \frac{\alpha / \gamma}{k} \text { or its powers }\right) .
\end{aligned}
$$

The first term dominates in the limit $\frac{\alpha / \gamma}{k} \rightarrow 0$.

$$
\Rightarrow \lim _{\frac{\alpha / \gamma}{k} \rightarrow 0} x_{s s}=\frac{\alpha}{\gamma}
$$




\section{Supplementary Figure 6.}

Flowchart for computing $Q_{x s s}$.

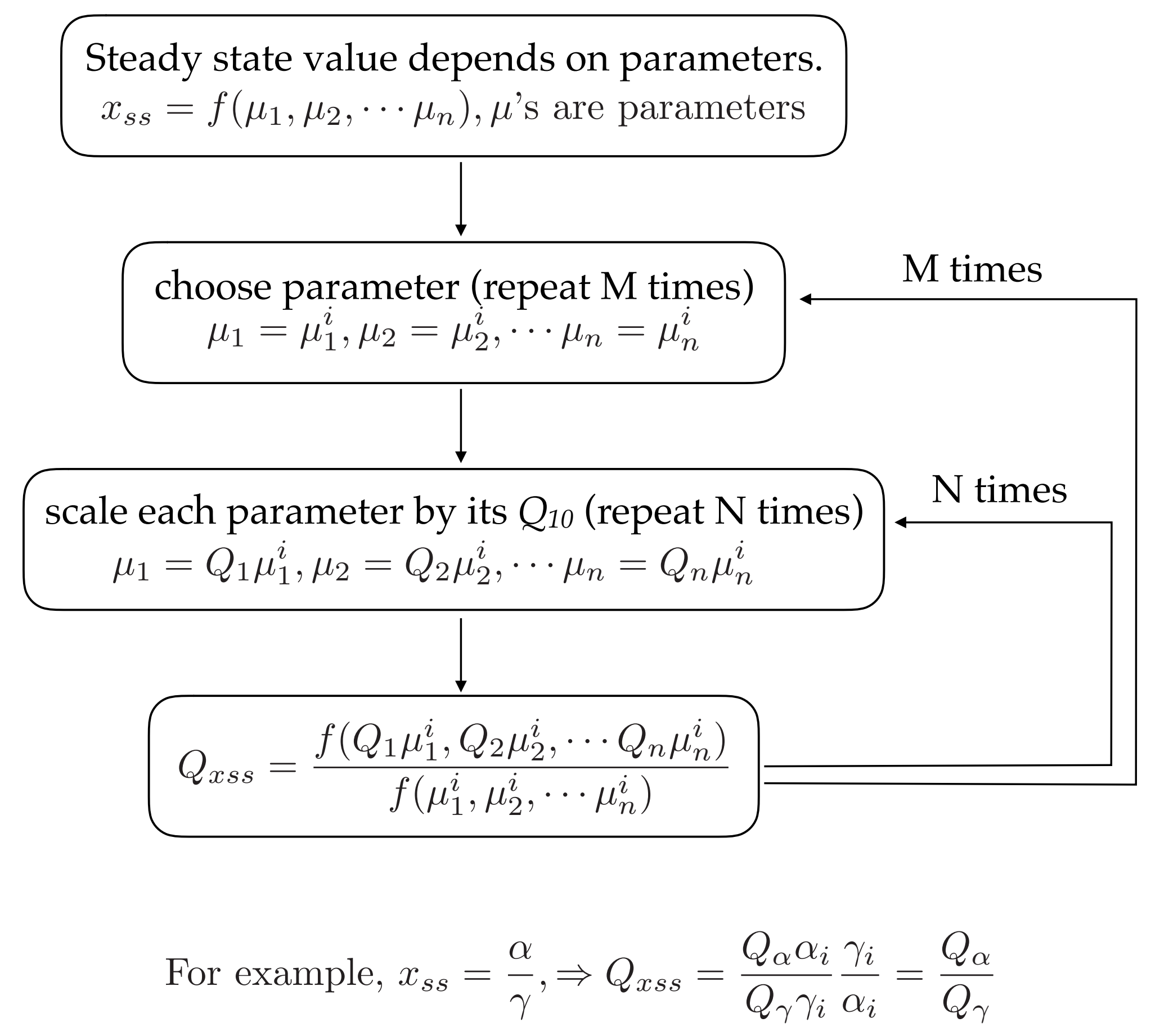




\section{Supplementary Figure 7.}

Computational assessment of the negative feedback loop for degradation rate $=\mathbf{1 0} / \mathrm{h}$. Captions are same as in Figure 4a-f.

(a)
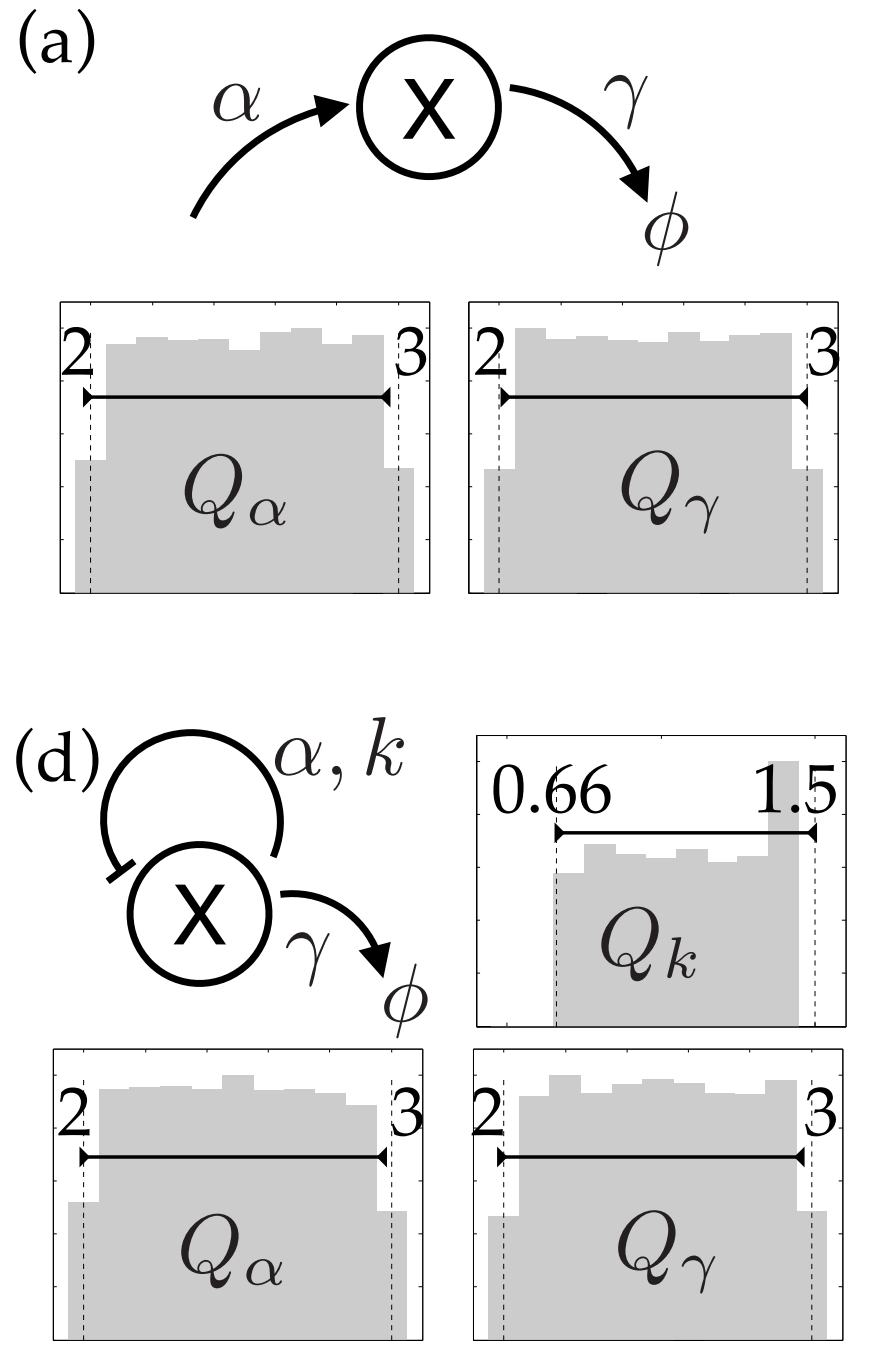

(b)

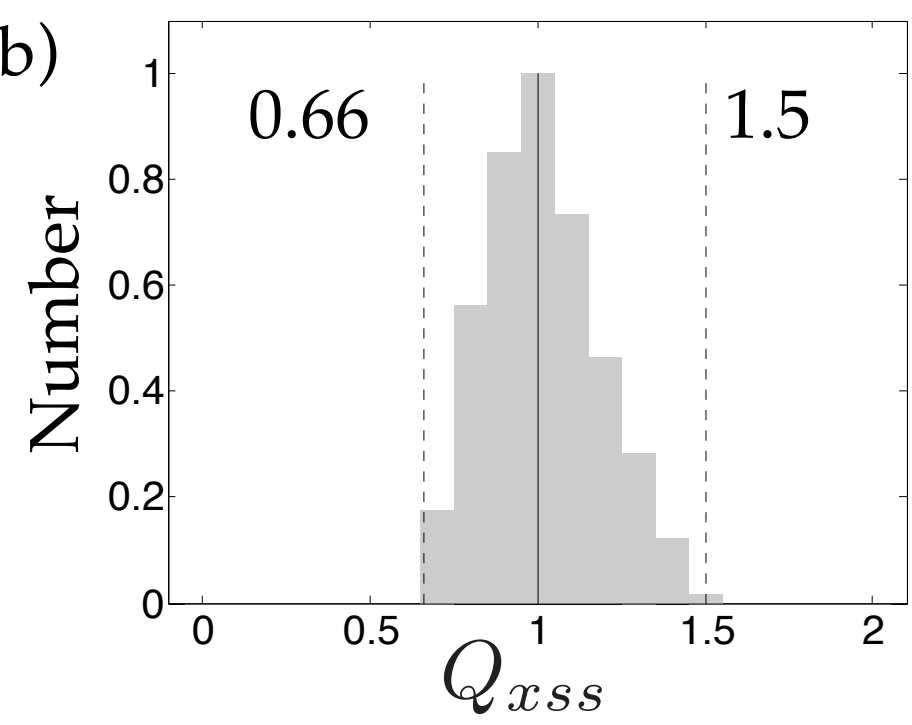

(e)

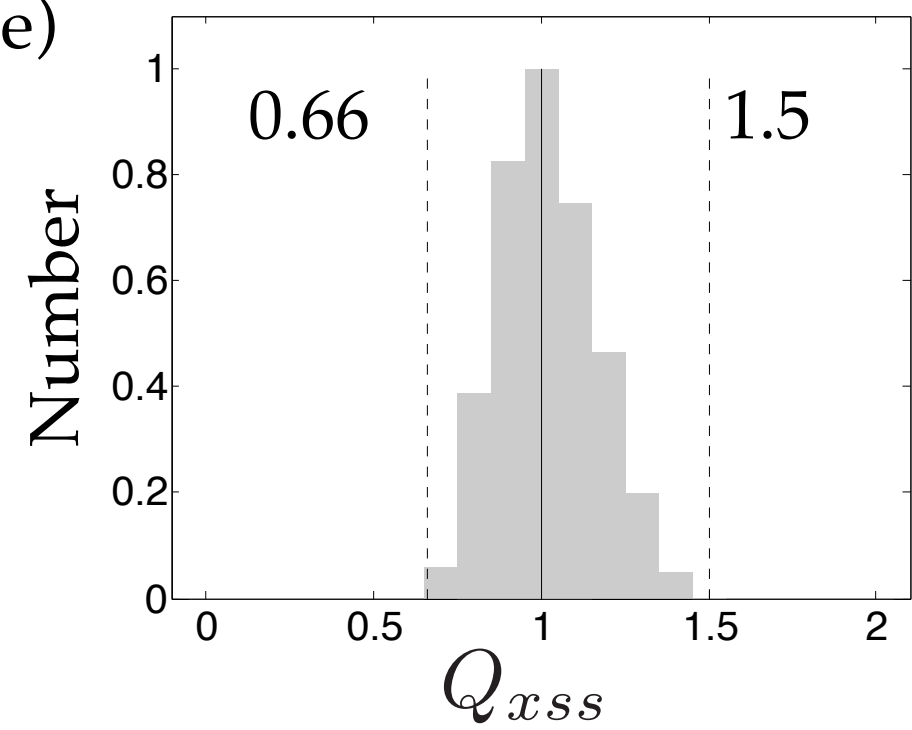

(c)

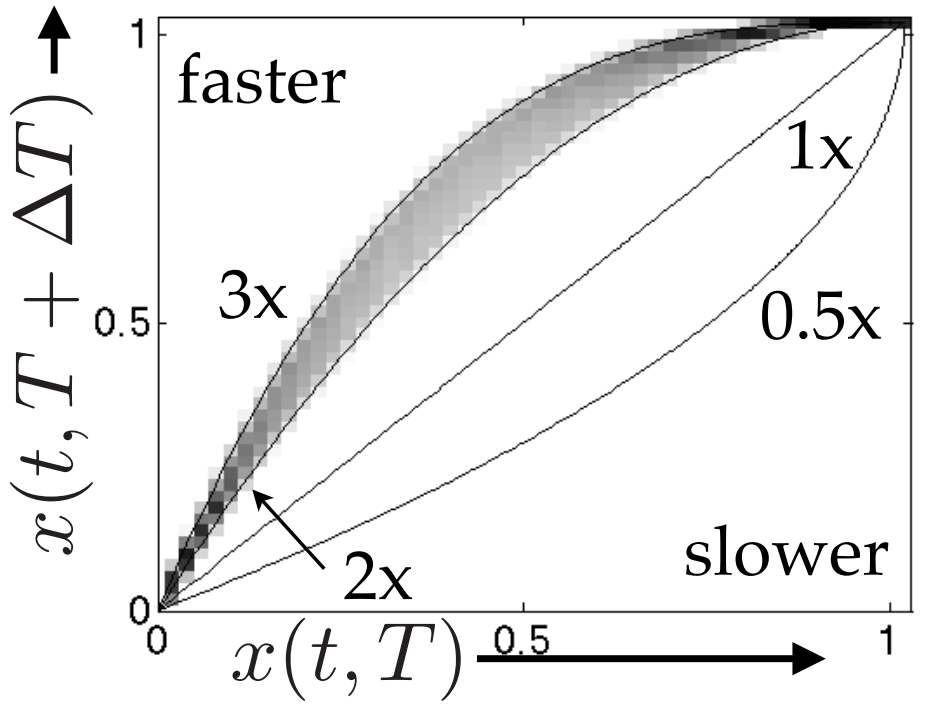

(f)

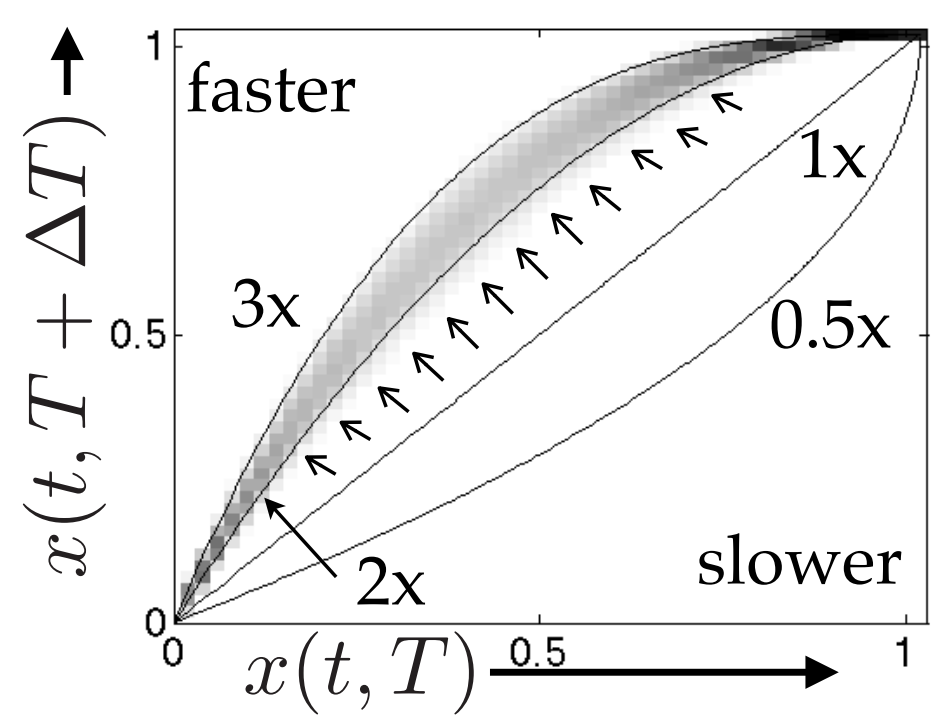




\section{Supplementary Figure 8.}

Computational assessment of the negative feedback loop for degradation rate $=\mathbf{0 . 1} / \mathbf{h}$. Captions are same as in Figure $4 \mathrm{a}-\mathrm{f}$.

(a)
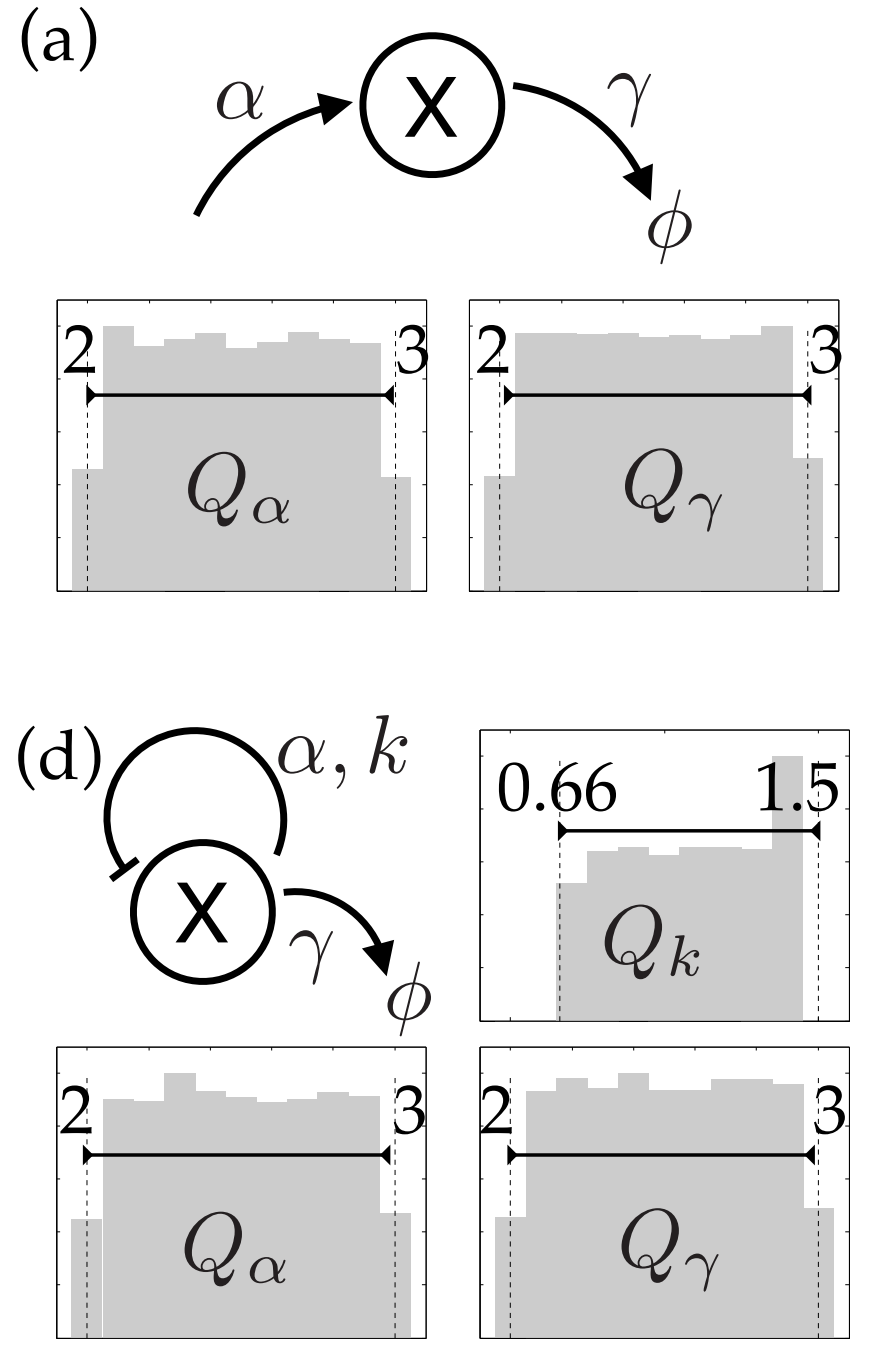

(b)

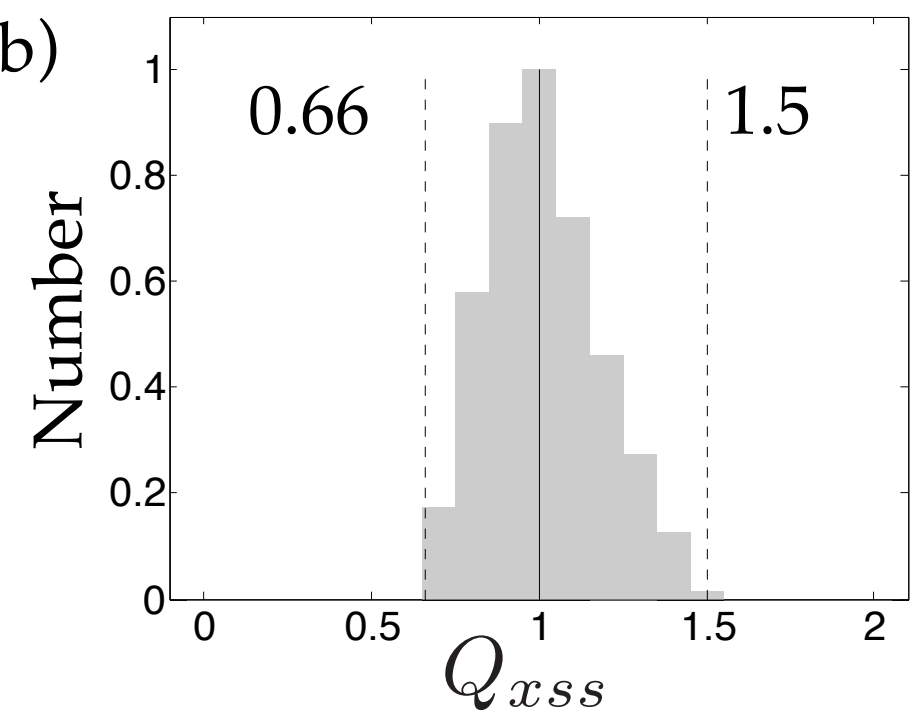

(e)

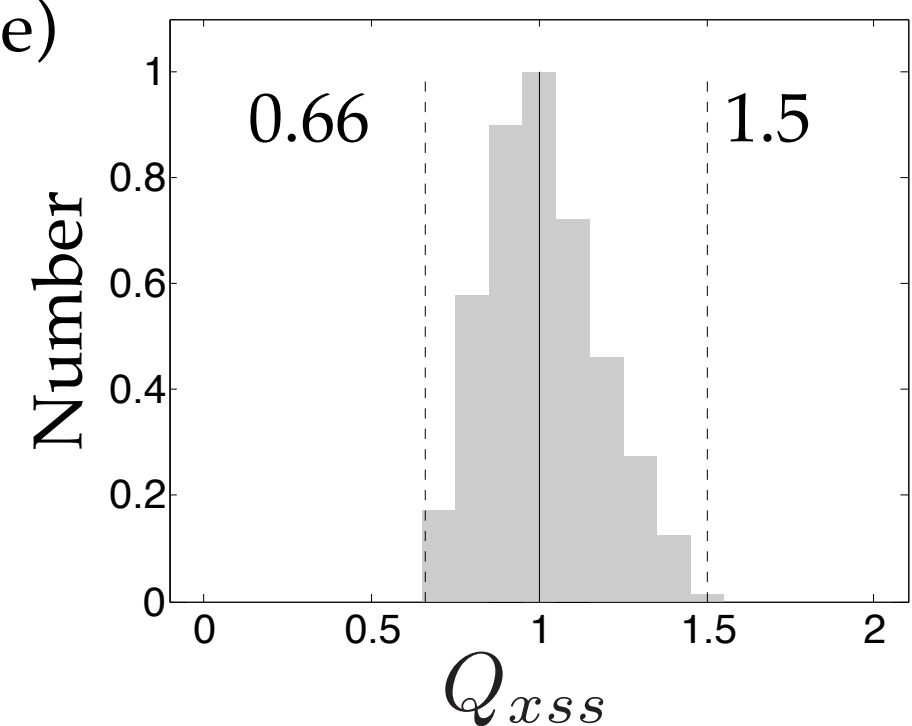

(c)

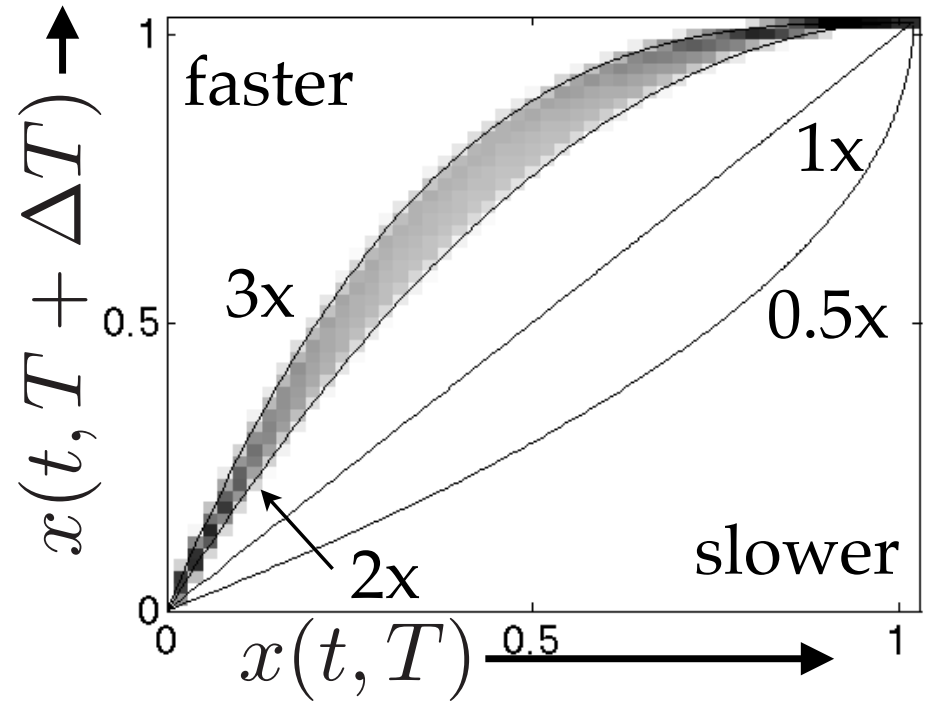

(f)

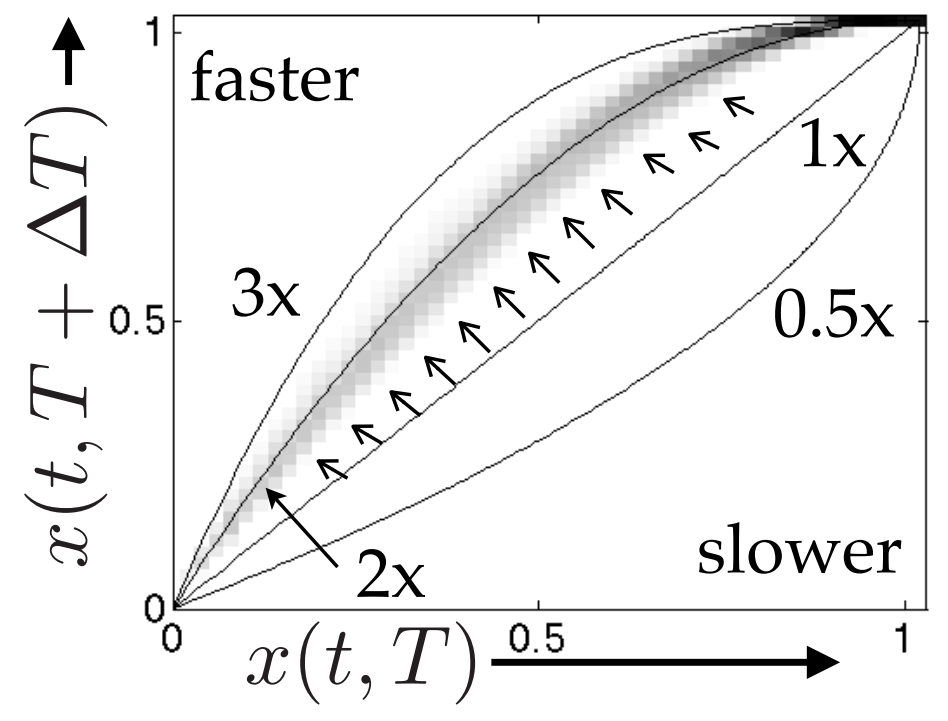




\section{Supplementary Figure 9.}

Computational assessment of the feedforward loop for degradation rate $=\mathbf{1 0} / \mathbf{h}$. Captions are same as in Figure $5 a-e$.
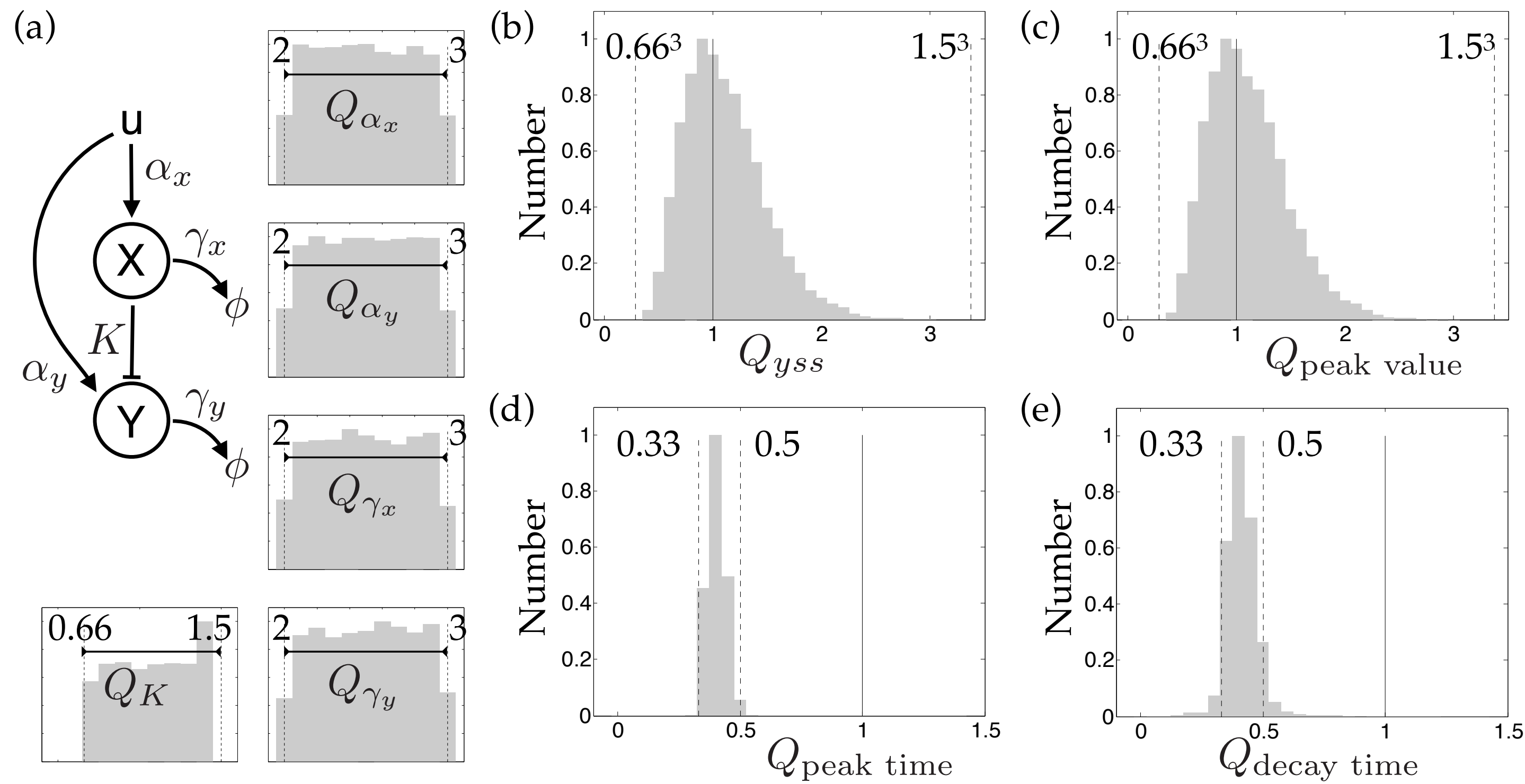


\section{Supplementary Figure 10.}

Computational assessment of the feedforward loop for degradation rate $=\mathbf{0 . 1} / \mathbf{h}$. Captions are same as in Figure 5 a-e.
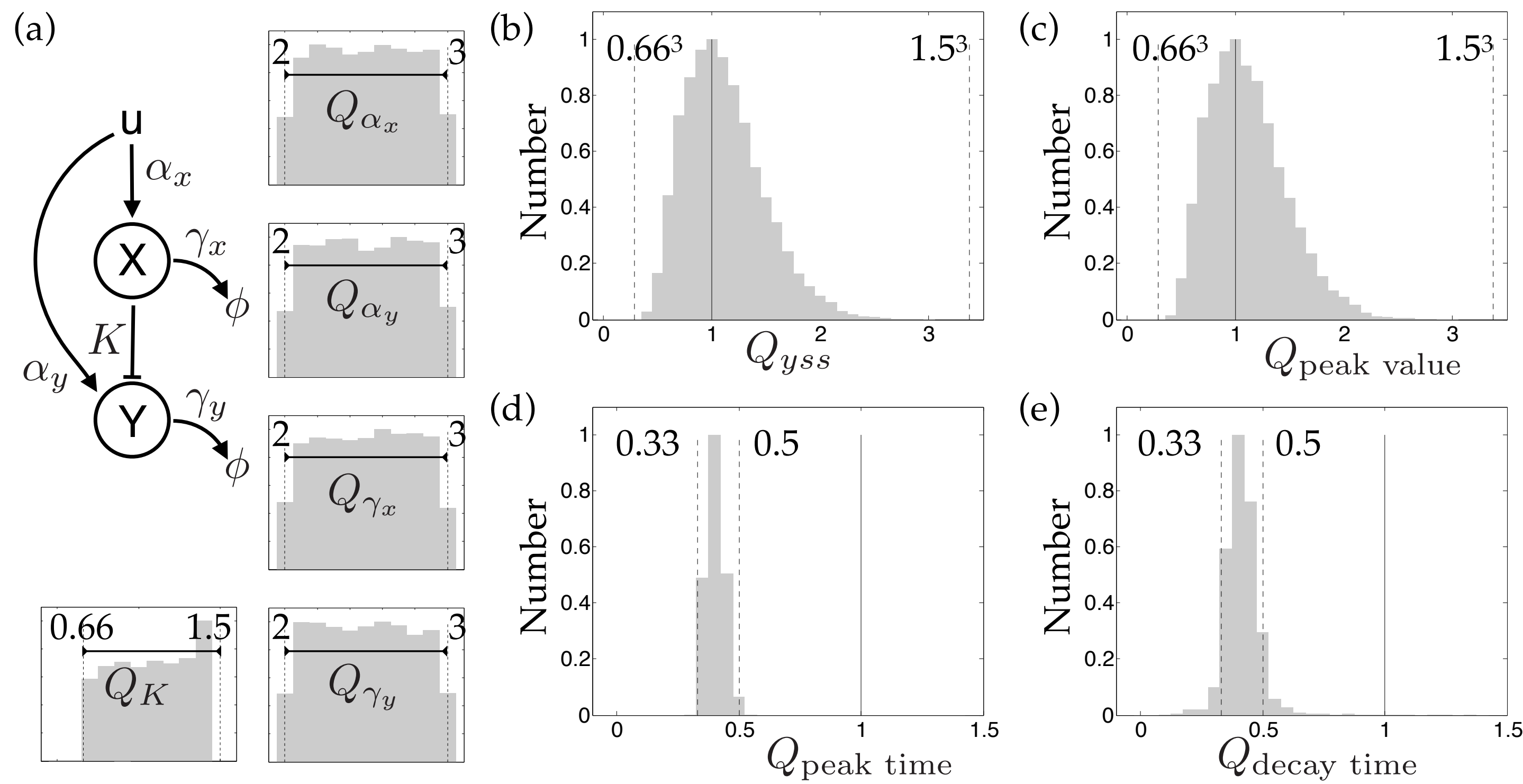


\section{Supplementary Figure 11.}

Computational assessment of an incoherent feedforward loop model with Hill-type gene regulation function. Equations are stated below. Same parameters as in the main text except that $K_{u}=100 \mathrm{nM}$ and $K_{x}=1 \mathrm{nM}$. These were chosen as not all parameter sets gave trajectories that pulsed (had a maximum). Units of $u$ are in $\mathrm{nM}$ for consistency.

$$
\begin{aligned}
& \frac{d x}{d t}=\alpha_{x} \frac{u / K_{u}}{1+u / K_{u}}-\gamma_{x} x, \\
& \frac{d y}{d t}=\alpha_{y} \frac{u / K_{u}}{1+u / K_{u}} \frac{1}{1+x / K_{x}}-\gamma_{y} y .
\end{aligned}
$$

Captions are same as in Figure 5a-e. Those parameters that pulsed were analysed for figure panels b-e.

(a)
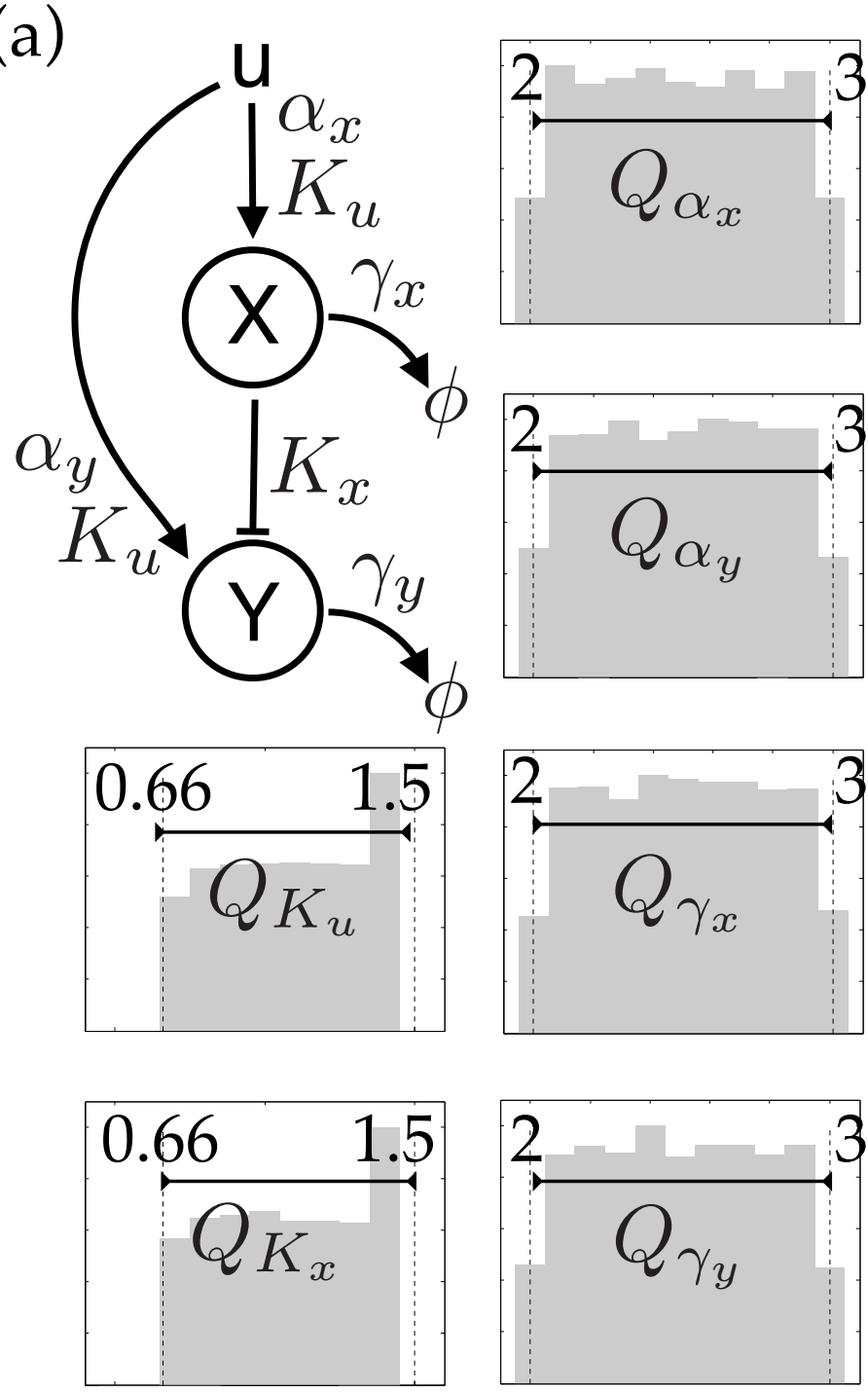
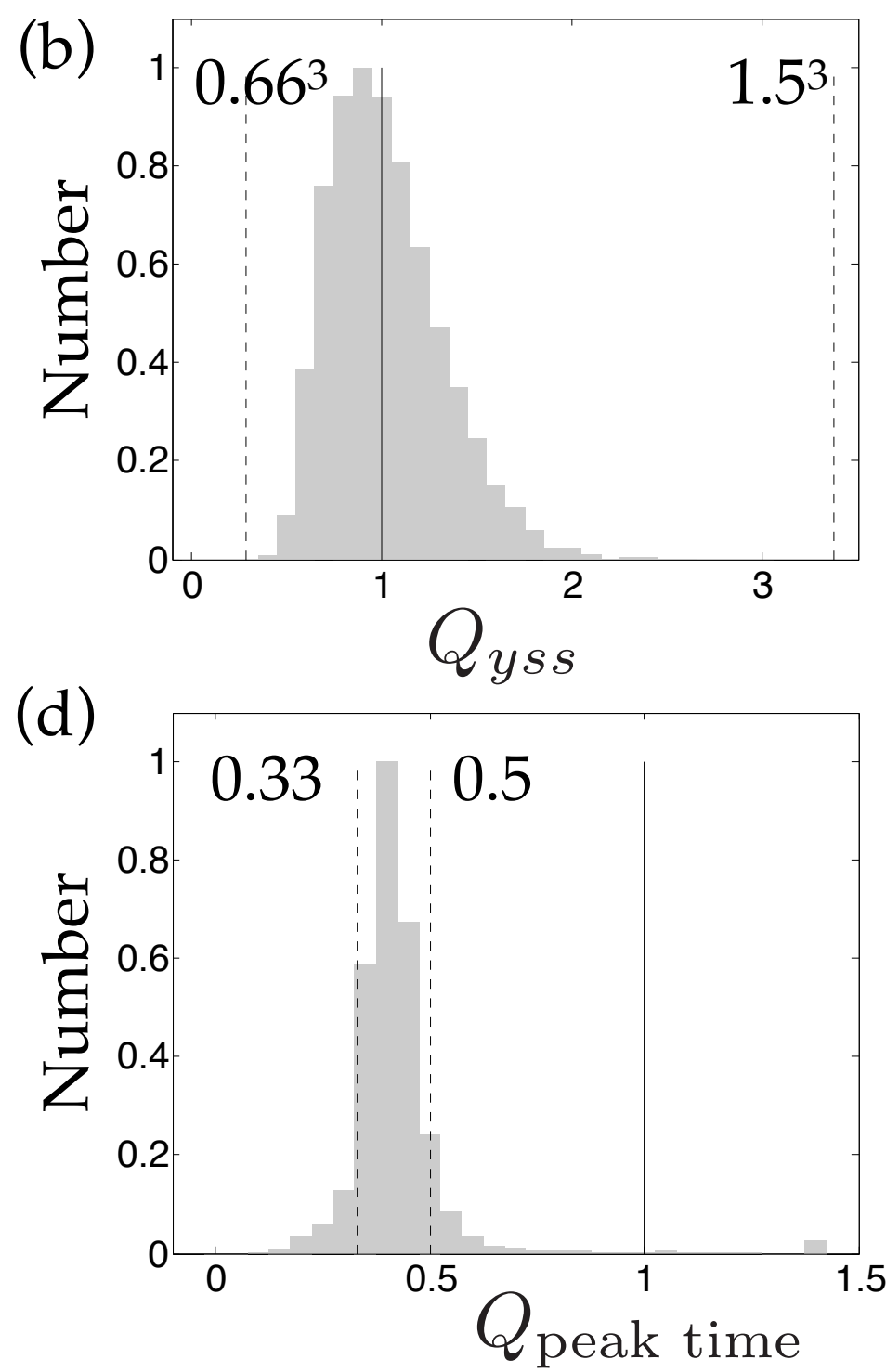
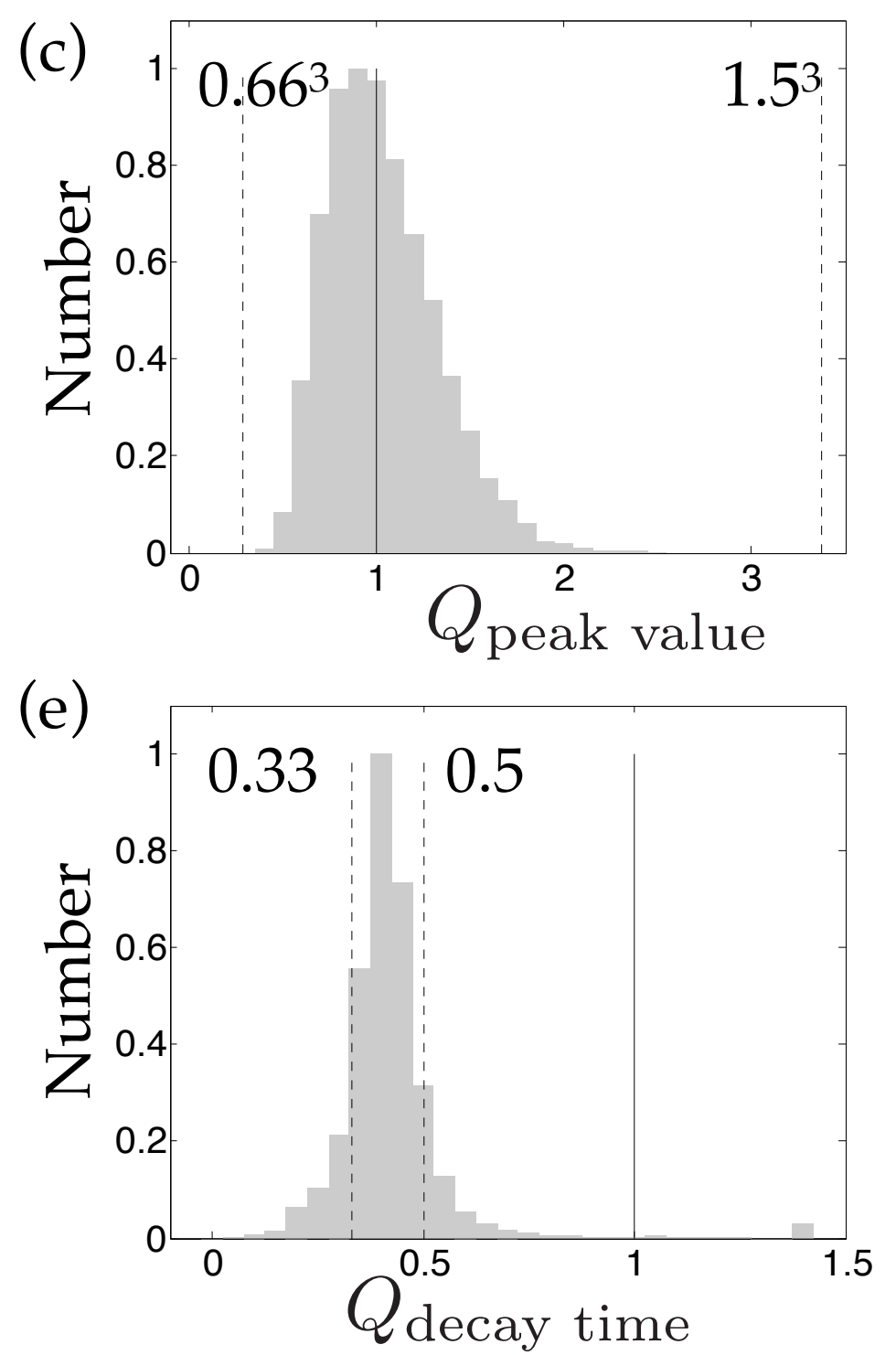


\section{Supplementary Figure 12.}

Computational assessment of a coherent feedforward loop model. Equations are stated below. (a) Schematic of a feedforward loop. Histograms show the assumed temperature coefficients of the parameters in the computations. (b) Histograms of the computed temperature coefficient of the delay time, computed as the difference in the times taken to reach half the steady state value of a coherent feedforward loop and a no feedforward circuit. Same parameters as in the Figure 5 of the main text except $K_{x}=100 \mathrm{nM}$.

$$
\begin{aligned}
& \frac{d x}{d t}=\alpha_{x} u-\gamma_{x} x, \\
& \frac{d y}{d t}=\alpha_{y} u \frac{x}{K_{x}}-\gamma_{y} y .
\end{aligned}
$$

(a)
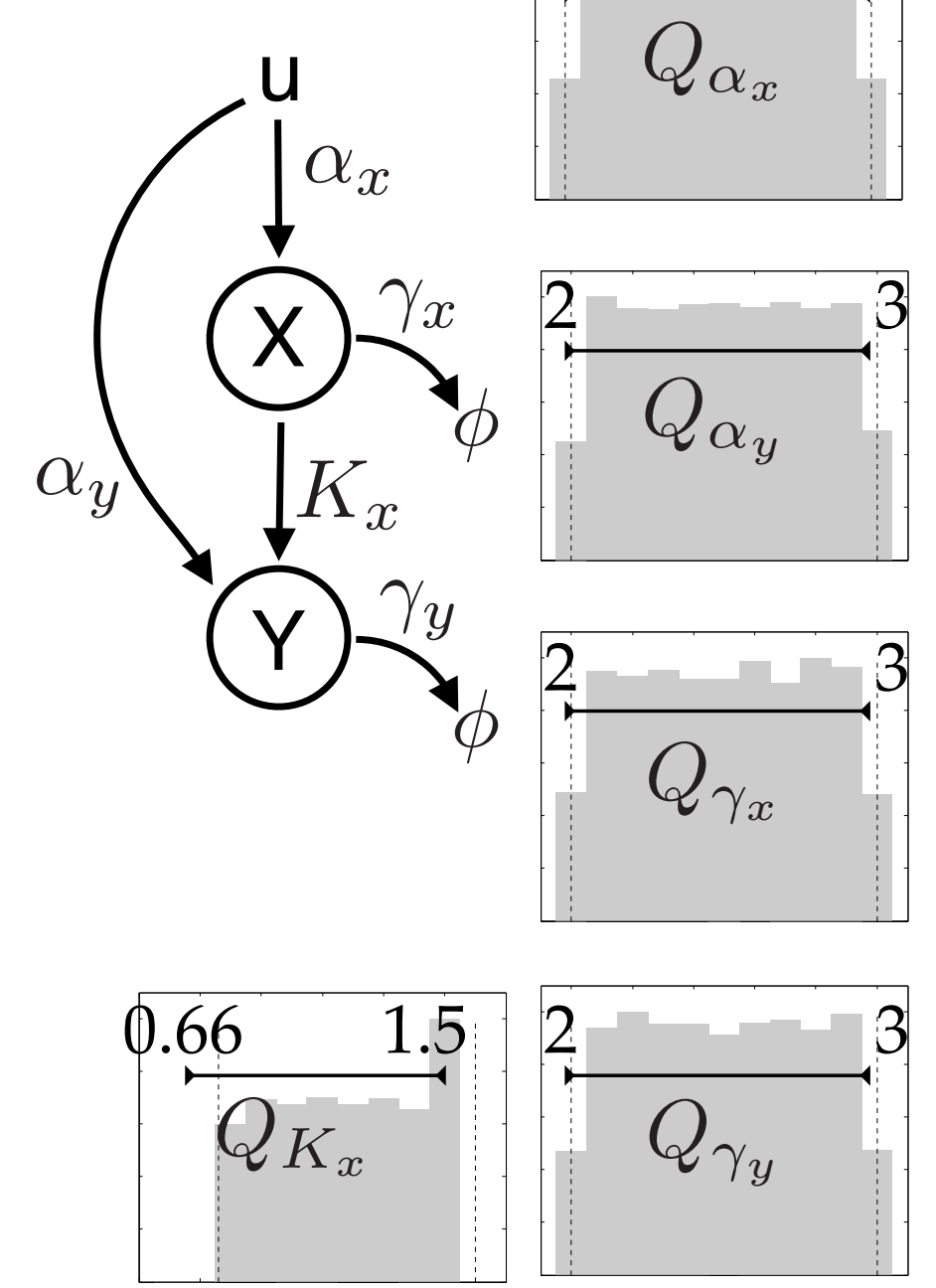

(b)

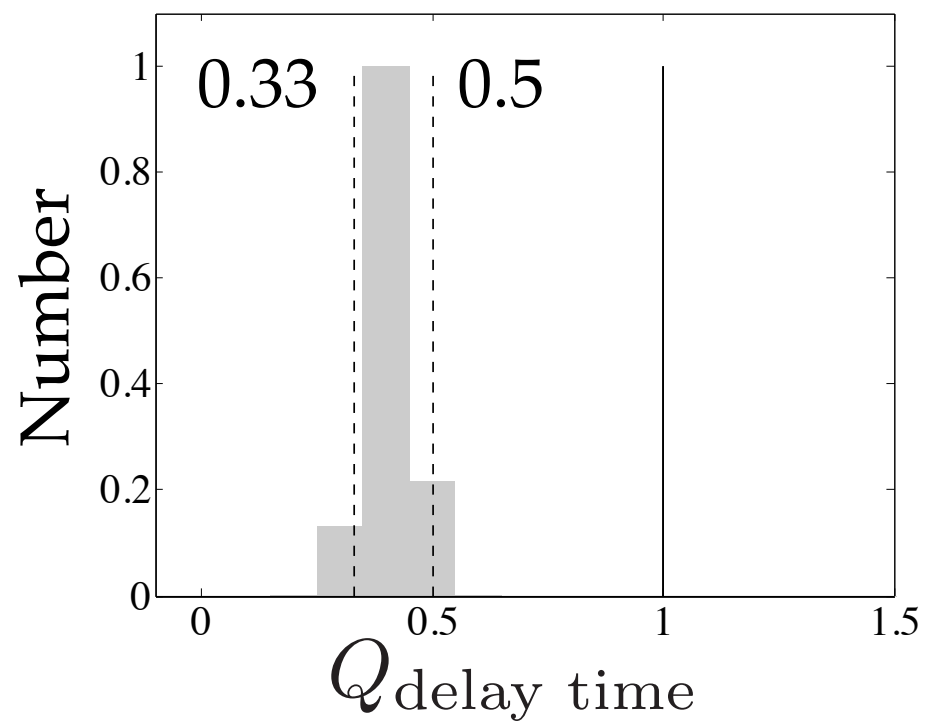




\section{Supplementary Figure 13.}

Computational assessment of a coherent feedforward loop model with Hill-type gene regulation function. Equations are stated below. (a) Schematic of a feedforward loop. Histograms show the assumed temperature coefficients of the parameters in the computations. (b) Histograms of the computed temperature coefficient of the delay time, computed as the difference in the times taken to reach half the steady state value of a coherent feedforward loop and a no feedforward circuit. Same parameters as in the Figure 5 of the main text except $K_{x}=100 \mathrm{nM}=K_{u}$.

$$
\begin{aligned}
& \frac{d x}{d t}=\alpha_{x} \frac{u / K_{u}}{1+u / K_{u}}-\gamma_{x} x, \\
& \frac{d y}{d t}=\alpha_{y} \frac{u / K_{u}}{1+u / K_{u}} \frac{x / K_{x}}{1+x / K_{x}}-\gamma_{y} y .
\end{aligned}
$$

(a)
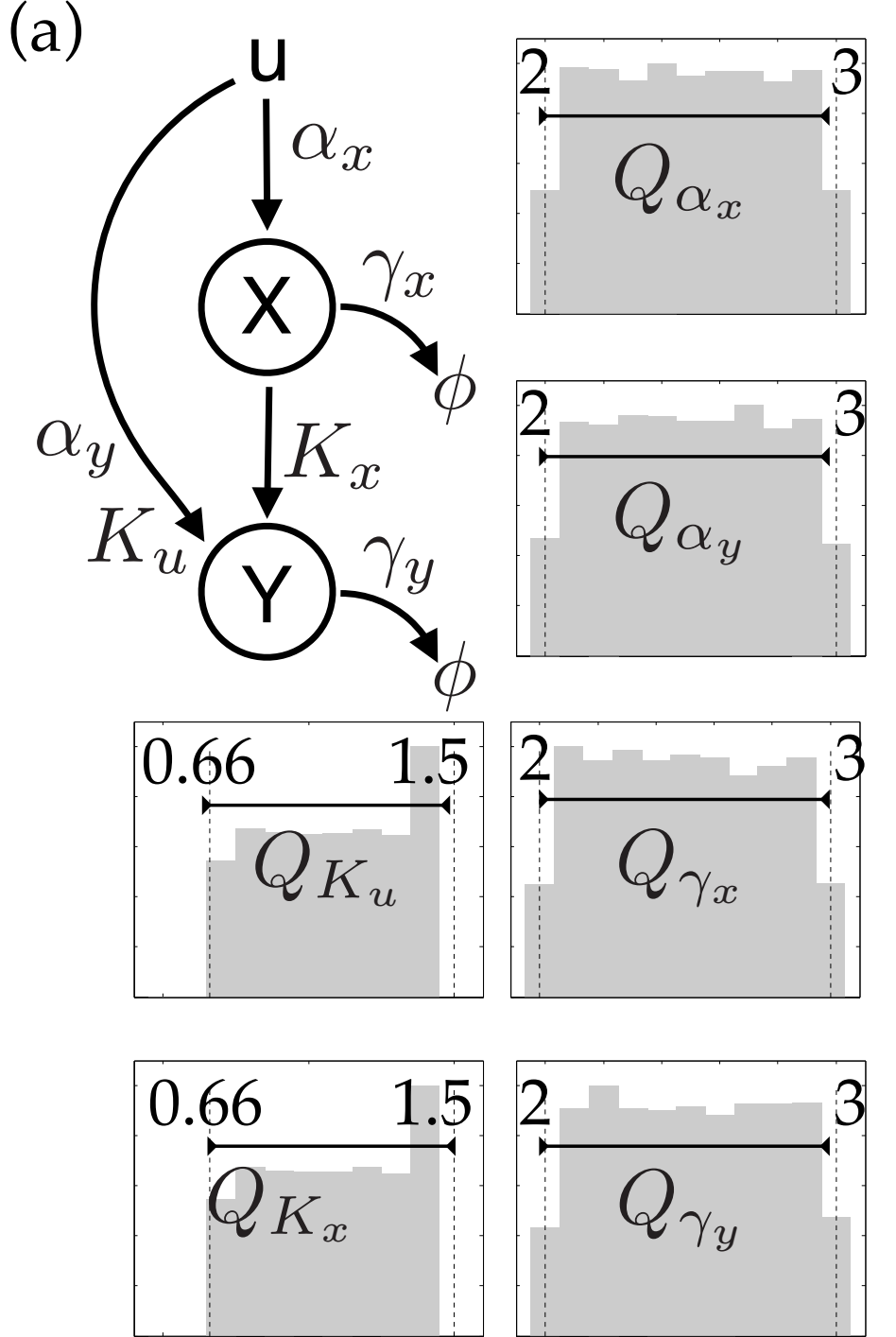

(b)

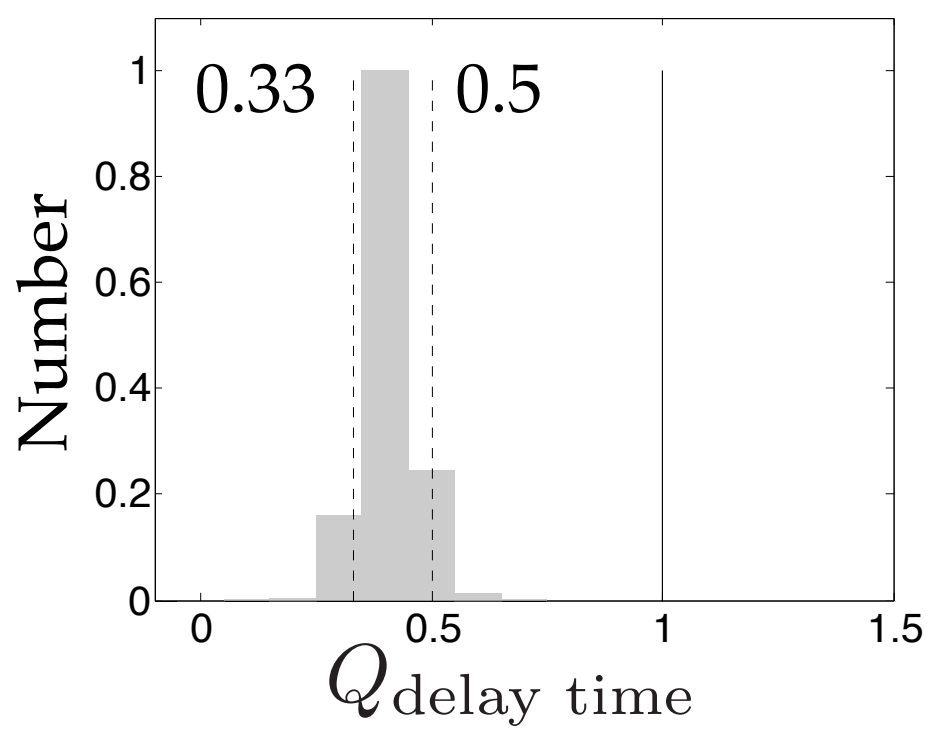




\section{Supplementary Figure 14.}

Growth rate of cells with the negative feedback circuit. (a), (b), and (c) show the growth rate computed from a first difference of the optical densities shown in Figures $2 \mathrm{e}, 2 \mathrm{f}$ and $2 \mathrm{~g}$, respectively.
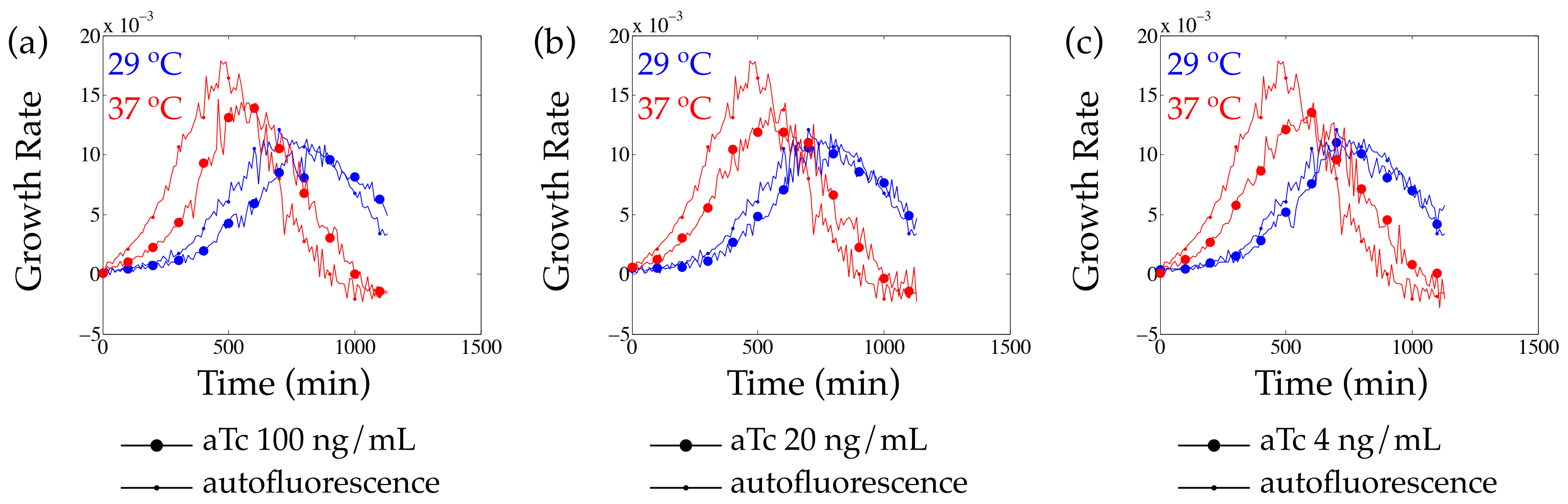


\section{Supplementary Figure 15.}

Correlation plot of trajectories of negative feedback circuit inside cells. (a) aTc $=100 \mathrm{ng} / \mathrm{mL}$, (b) aTc $=20 \mathrm{ng} / \mathrm{mL}$, (c) aTc $=4 \mathrm{ng} / \mathrm{mL}$. Green line represents the

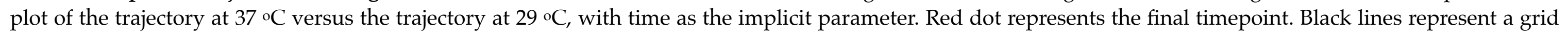
of exponential trajectories that are identical (diagonal), or faster by indicated amounts.

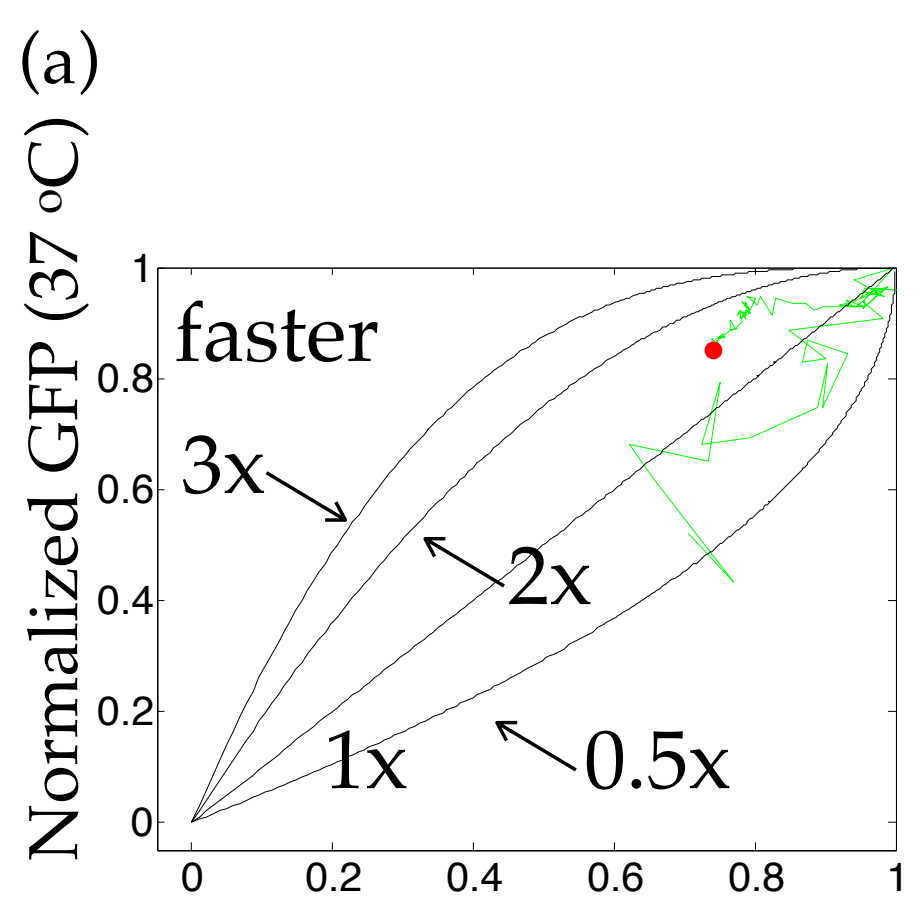

Normalized GFP $\left(29^{\circ} \mathrm{C}\right)$ (b)

তิ

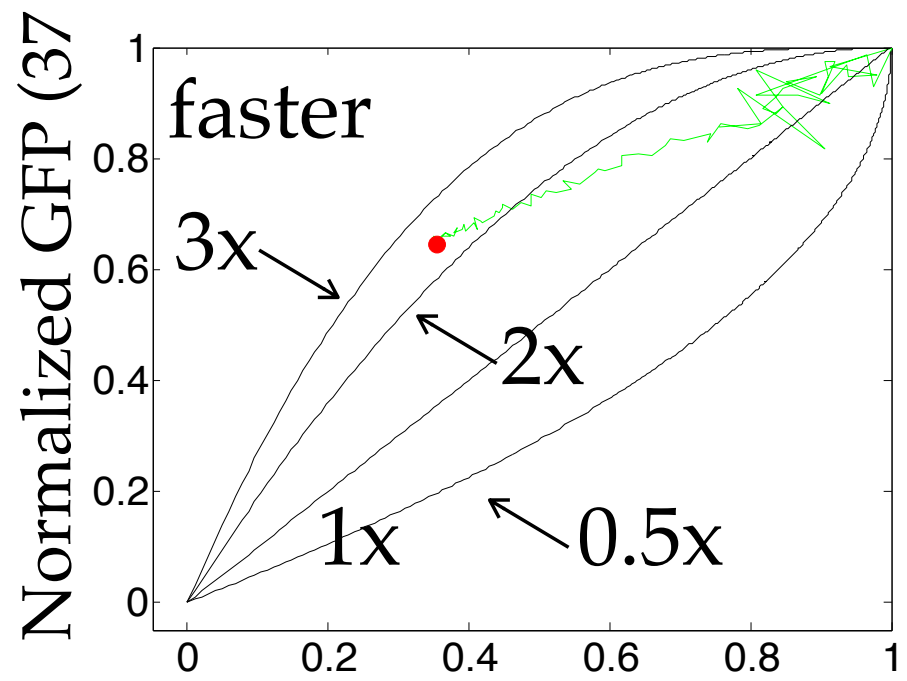

Normalized GFP $\left(29{ }^{\circ} \mathrm{C}\right)$ (c)

ชิ

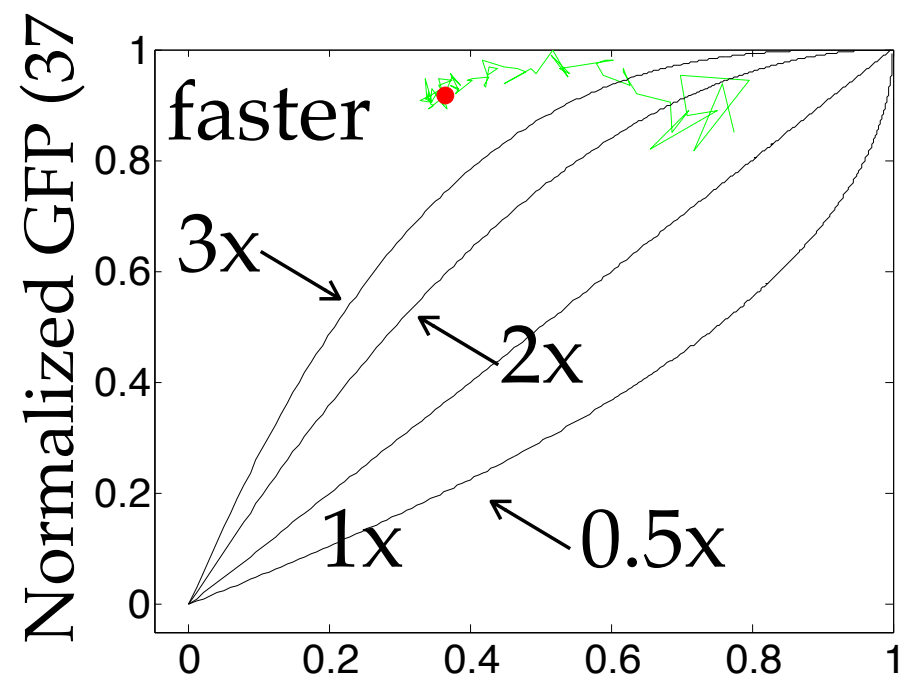

Normalized GFP $\left(29{ }^{\circ} \mathrm{C}\right)$ 


\section{Supplementary Figure 16.}

Response of the negative feedback plasmid at different concentrations in the cell-free system. Solid colored lines indicate the response at labeled concentrations. Black dashed line is background.

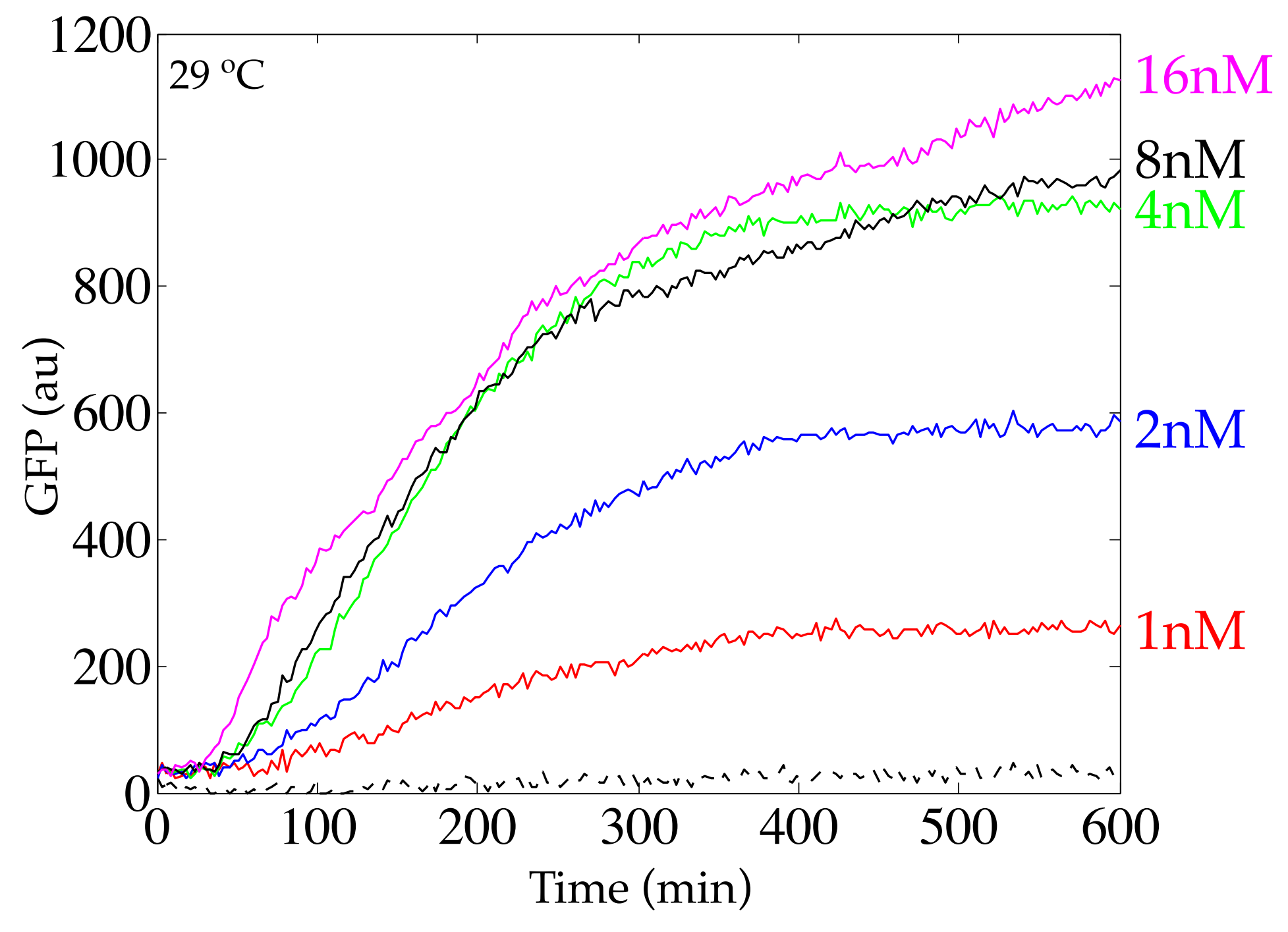




\section{Supplementary Figure 17.}

Figure 6a-c with background showing all individual traces.

(a)

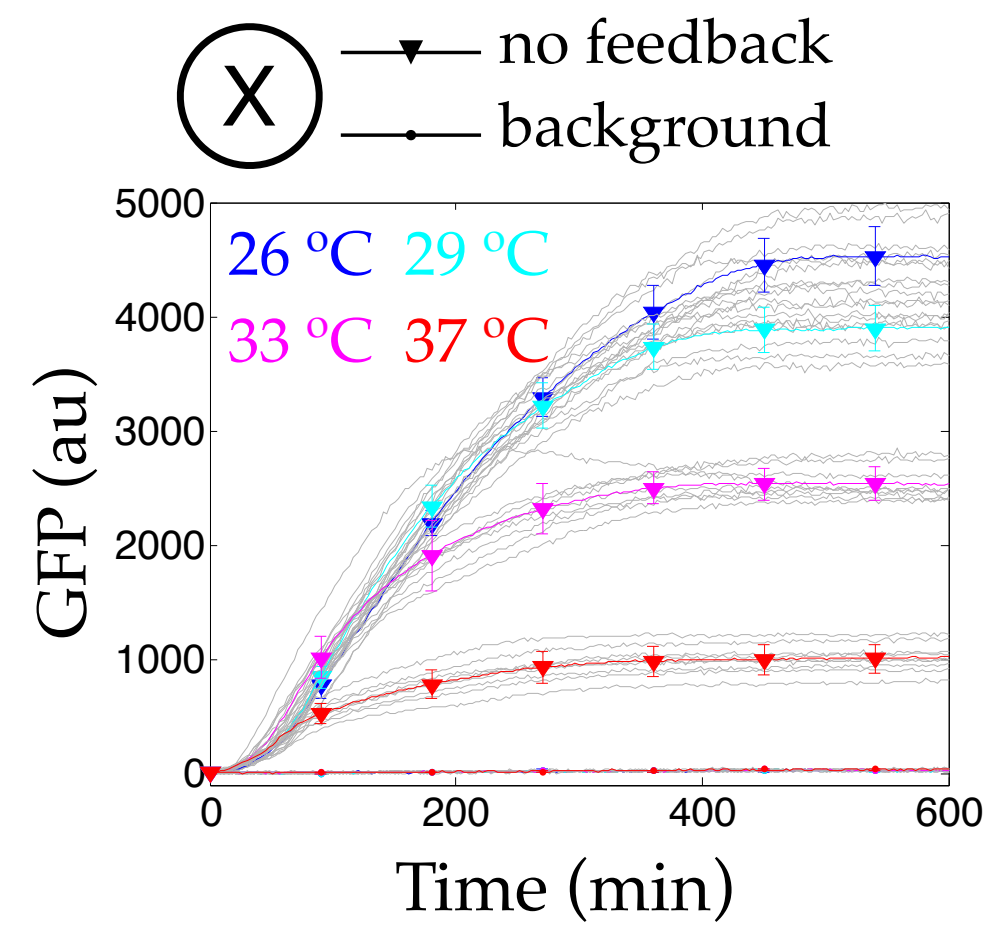

(b)
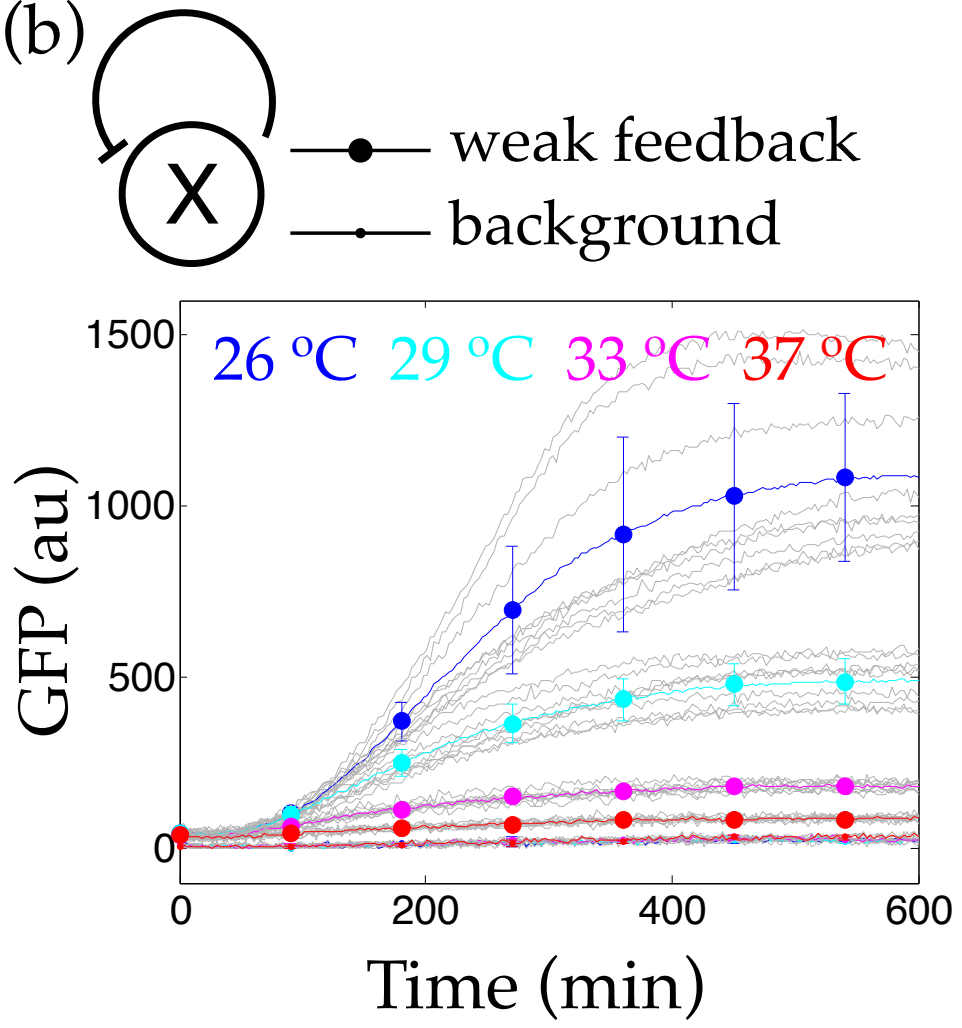

(c)
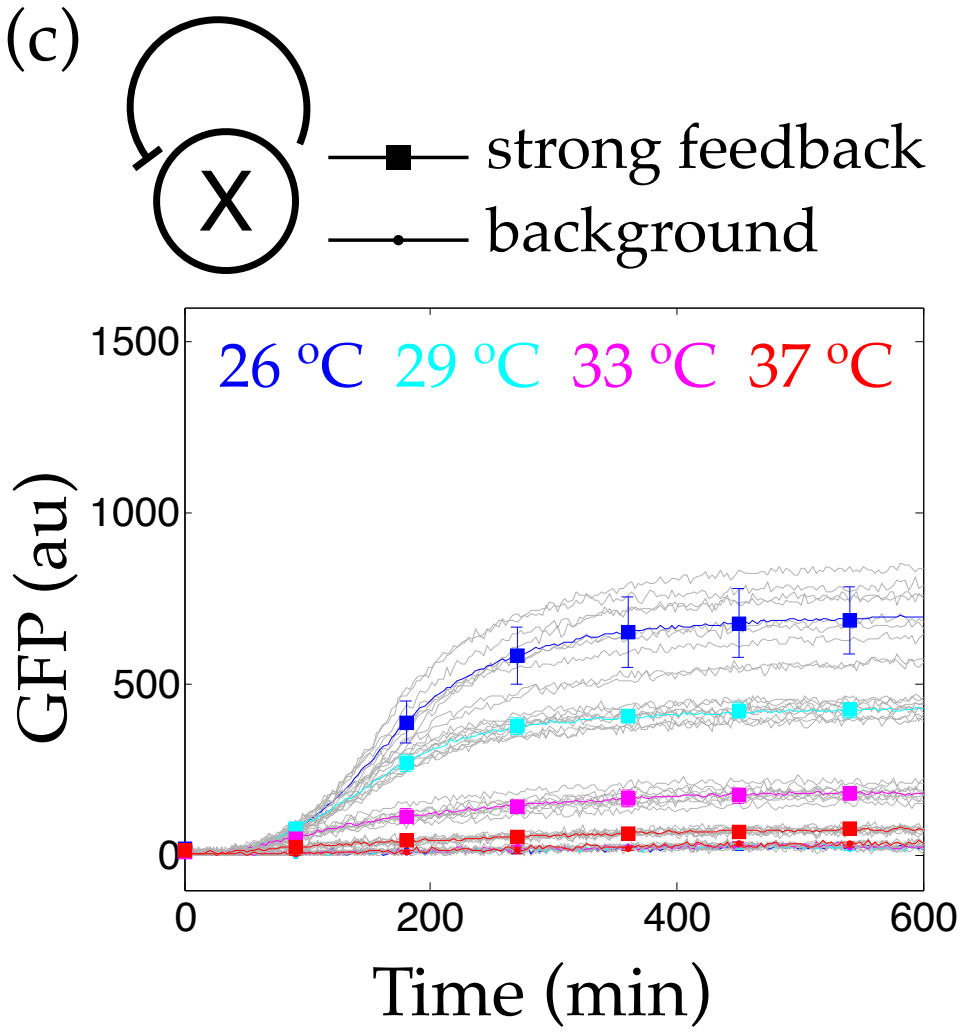


\section{Supplementary Figure 18.}

Smoothened and bolder version of the lines shown in Figures 6e and 6f. Smoothening was done using the MATLAB 'smooth' function with default options.
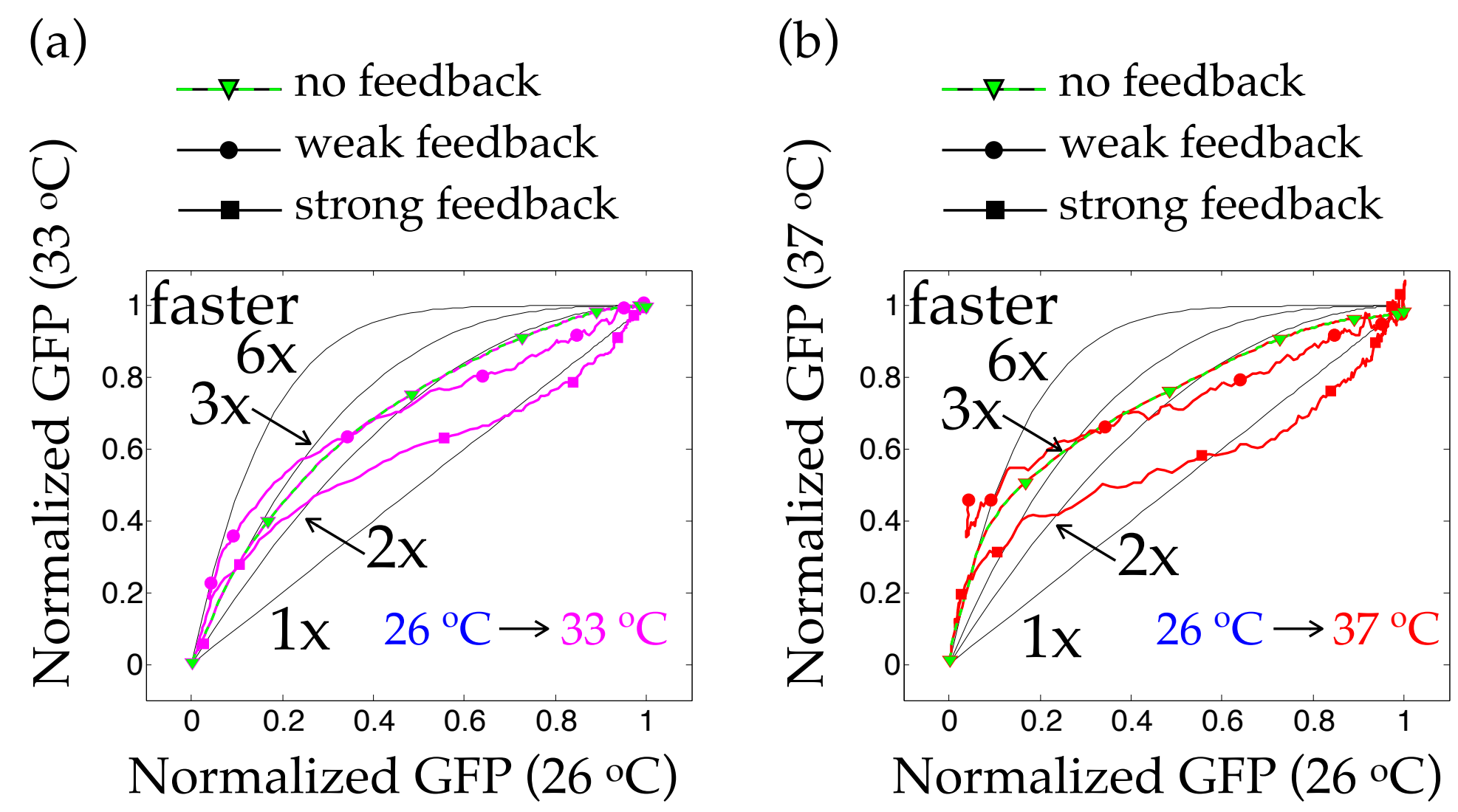


\section{Supplementary Figure 19.}

Computational assessment of a model of negative feedback loop in a cell-free system with resource limitation. Schematic, mathematical model, parameter $Q_{10}$, and plots of transient response normalized to end-point before and after a temperature shift for (a) No feedback circuit, and (b) Negative feedback circuit. Model parameter values are same as in the main text. Resource $(\beta)$ was modelled as decaying in a first-order fashion from an initial value of 1 . The simulation was run for $\mathrm{t}=10 \mathrm{~h}$.

(a)

$$
\begin{aligned}
& \alpha \frac{d \beta}{d t}=-\gamma \beta \\
& \frac{d x}{d t}=\alpha \beta
\end{aligned}
$$
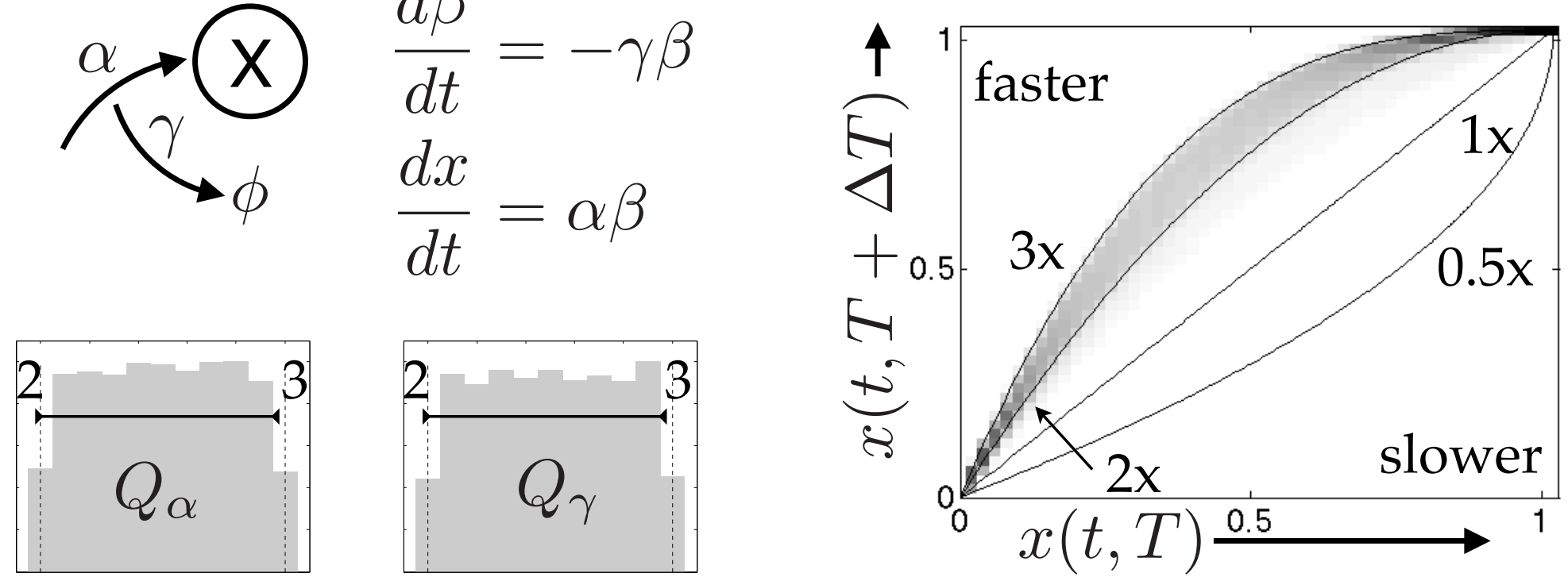

(b)

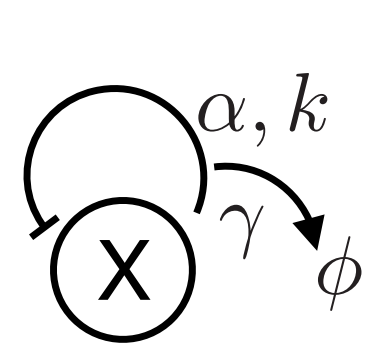

$$
\begin{aligned}
& \frac{d \beta}{d t}=-\gamma \beta \\
& \frac{d x}{d t}=\frac{\alpha \beta}{1+x / k}
\end{aligned}
$$
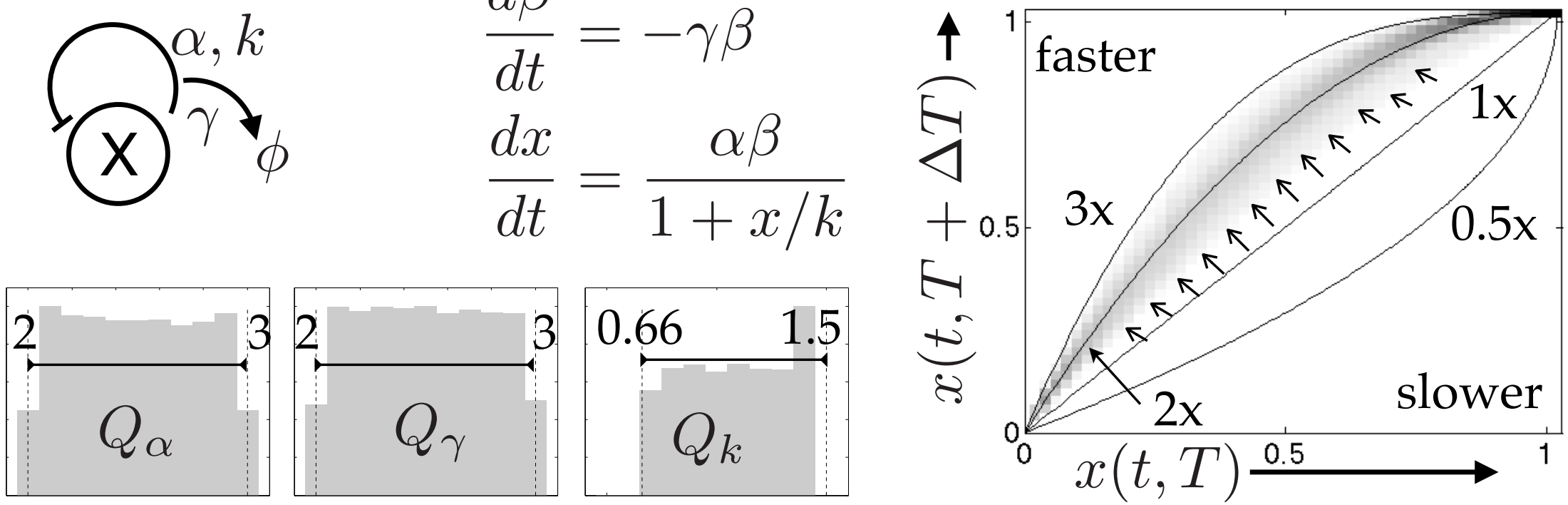